\title{
« En ville, les Africains sophistiqués ne mangent pas de ces plats-là »
}

\author{
Une histoire sociale de la consommation de petites céréales à Bulawayo (Rhodésie \\ du Sud, années 1920-1950)
}

\author{
Bryan Kauma et Sandra Swart
}

Traduit de I'anglais par Gilles Baro, Sophie Dulucq et Camille Evrard

\begin{abstract}
Citer cet article : Kauma Brian, Swart Sandra (2021), «"En ville, les Africains sophistiqués ne mangent pas de ces plats-là". Une histoire sociale de la consommation de petites céréales à Bulawayo (Rhodésie du Sud, années 1920-1950) », Revue d'Histoire Contemporaine de l'Afrique, n², 89-116, en ligne. URL :

https://oap.unige.ch/journals/rhca/article/view/02kaumaswart
\end{abstract}

Mise en ligne : $1^{\text {er }}$ octobre 2021.

DOI : https://doi.org/ 10.51185/journals/rhca.2021.e554

\section{Résumé}

Fondée en 1840, la ville de Bulawayo témoigne du développement urbain et de l'évolution des cultures alimentaires en Afrique australe et en Rhodésie du Sud (actuel Zimbabwe). Au temps du Gouvernement Responsable de 1923, la ville compte une population très diverse et s'impose non seulement comme le centre économique de la colonie britannique naissante, mais aussi comme le lieu emblématique des relations complexes entre Noirs et Blancs qui caractérisent la période coloniale. En s'appuyant sur l'histoire de la cuisine et des habitudes alimentaires liées aux petites céréales africaines - sorgho, millet et rapoko-, cet article retrace le développement de pratiques alimentaires nouvelles à Bulawayo. Basé sur les Archives nationales du Zimbabwe et I'historiographie existante, il a recours à I'histoire de ces petites céréales dans l'alimentation des citadins pour repenser certains aspects des relations socio-politiques entre Noirs et Blancs dans les cultures urbaines africaines, du début des années 1920 à l'aube de la Fédération des Rhodésies et du Nyassaland en 1953. Contribuant à une historiographie des villes africaines en plein essor, cet article démontre aussi comment, à l'époque coloniale, I'histoire des relations sociales et des changements de consommation à Bulawayo peut être analysée au prisme de l'estomac.

Mots-clés : petites céréales ; maïs ; modèles culinaires; histoire de l'alimentation ; colonialisme ; culture urbaine africaine ; Bulawayo ; Rhodésie du sud ; Zimbabwe 
Les mots suivants résonnent : "Kana ukandibikira sadza ne broccoli, ndinoti aaah ndakaguta » ("Si tu me cuisines de la sadza avec du brocoli, je vais dire que je n'ai pas faim »)1. Provoquant de vives acclamations et des rires parmi les 70000 militants et les responsables nationaux du parti, lors d'un meeting politique en septembre 2017, cette saillie de Robert Gabriel Mugabe, alors président du Zimbabwe, soulève une série de questions sur les modes de consommation de la nourriture de base dans le pays : la sadza². Existe-t-il une manière " correcte », et « incorrecte », d'en manger ? Y a-t-il une façon politiquement incorrecte - une façon «non africaine »- de la consommer ? Et si c'est le cas, quelle est-elle et comment a-telle évolué ? À qui doit-on cette évolution et quel en est le motif ? Pourquoi cet aliment est-il devenu politique et, finalement, qu'est-ce qui a contribué à ces mutations ?

Plus largement, nous allons explorer dans cet article I'histoire sociale et politique des pratiques alimentaires et culinaires au sein des familles noires de Bulawayo, deuxième ville de Rhodésie du Sud (Zimbabwe), en retraçant l'histoire des petites céréales que sont le sorgho, le millet et le rapoko ${ }^{3}$. Nous analysons les principaux changements dans les pratiques alimentaires durant la période coloniale, du début des années 1920 à l'aube de l'Union Fédérale entre les Rhodésies du Nord et du Sud et le Nyassaland, en 1953. Cet article a pour ambition de contribuer au débat historiographique sur les liens entre alimentation et société urbaine : nous concentrons notre attention sur la façon dont les petites céréales sont consommées par différents groupes d'Africains noirs à Bulawayo, et sur la manière dont les différents facteurs sociaux, environnementaux et politiques ont façonné l'alimentation et les coutumes culinaires au fil du temps. Nous soutenons l'idée que la cuisine à base de petites céréales africaines et les techniques alimentaires sont alors protéiformes, diversement structurées par des choix d'acteurs africains confrontés à des mutations rapides sur les plans socio-politique et économique. Au-delà des lignes de clivage traditionnelles, nous démontrons que, si les Africains ordinaires ont parfois été contraints d'adopter de nouvelles idées sur les aliments et sur les façons de manger, ils se sont adaptés en réponse à des vicissitudes environnementales et économiques en constante évolution, en adaptant leurs anciens systèmes culinaires ou en en inventant de nouveaux.

Dans le cadre des débats historiographiques portant sur les efforts fournis des colonisateurs pour façonner les espaces urbains et les relations entre Africains et Blancs en ville, nous souhaitons montrer comment, entre 1924 et 1953, les habitudes alimentaires et culinaires des Africains en ce qui concerne les petites céréales ont été façonnées et transformées par une multitude de facteurs sociaux, politiques et économiques spécifiquement urbains. De même, nous voulons mettre en évidence le fait que la cuisine et l'alimentation ordinaires n'ont pas été soumises à de simples évolutions sociales, mais ont été affectées par

\footnotetext{
1 « Speech by President Mugabe at Zanu PF presidential youth interface rally Gweru, Midlands province, 1 er septembre 2017 », en ligne. URL: https://www.facebook.com/ZimbabweTodayLive/videos/mugabe-on-eating-sadza-and-broccoli-/1505362159628845/ (consulté le $1^{\mathrm{er}}$ août 2021). Le public rit et pousse des huées parce qu'au Zimbabwe, comme le montre cet article, un aliment tel que le brocoli est aujourd'hui encore considéré comme élitiste, réservé à quelques privilégiés, et n'est donc généralement pas vu comme un aliment qui puisse accompagner la sadza. De plus, dans une interview en mai 2013, la famille Mugabe a raconté comment le président s'était sevré d'un régime à base de maïs blanc pour des raisons diététiques, et son allusion à la sadza renvoie ici à la recette préparée à base de petites céréales.

2 La sadza est un porridge épais préparé à base de farine de maïs ou de petites céréales. Il est plutôt consommé chaud mais, comme le montre cet article, manger de la sadza froide est de plus en plus courant chez les mineurs noirs, dès les années 1930.

${ }^{3}$ Variété de millet (Eleusine caracana).
} 
des efforts politiques systématiques visant à créer une société urbaine idéale en Rhodésie du Sud. De nombreux anthropologues, ethnographes et sociologues spécialistes de l'alimentation se sont généralement concentrés sur quelques questions liées aux aliments, principalement sur les rites faisant appel à la nourriture, à sa signification, son goût, sa couleur, sa texture. Ils ont souvent étudié l'alimentation dans une démarche anhistorique.

Est-il possible de proposer une histoire différente de la cuisine africaine, au-delà de ces formes d'interprétation ? En centrant la réflexion sur la ville de Bulawayo (cartes 1 et 2), cette recherche explorera comment les habitudes alimentaires liées aux petites céréales ont évolué au fil du temps, afin de montrer que l'histoire de la nourriture offre aussi un récit sur la concurrence des espaces, sur l'hégémonie de certains aliments et sur l'agentivité (agency) des Africains en contexte urbain. II s'agira d'étudier comment de multiples facteurs socio-politiques ont affecté les habitudes alimentaires des Africains, modelant un aspect fondamental des modes de vie urbains. Nous verrons également comment les Africains ont réagi à ces transformations. En nous appuyant sur diverses sources primaires conservées aux Archives nationales du Zimbabwe (National Archives of Zimbabwe) ainsi que sur des entretiens oraux, nous montrerons que ces évolutions des habitudes alimentaires des familles noires ont non seulement influé sur les modes de consommation, mais aussi sur la façon dont les Africains se représentent le statut social.

Afin d'analyser ces dynamiques importantes, cet article propose une approche à la fois thématique et chronologique. Un bref bilan historiographique permettra de situer cette étude, offrant une contextualisation préalable permettant de retracer l'histoire des modèles alimentaires au Zimbabwe. On abordera ensuite la question des politiques liées à l'alimentation et à la consommation. Nous évoquerons les premières années dites du «Gouvernement responsable » (Responsible Government), qui conduisent à la promulgation de la Loi sur le contrôle du maïs (Maize Control Act) de 1930, en montrant comment, en matière agricole, certaines cultures sont alors devenues dominantes, et ont influé sur les pratiques alimentaires et le développement des petites céréales. Nous nous concentrons sur la période de mise en place de la législation sur le maïs - législation dont les effets sur les pratiques alimentaires sont considérables. L'introduction de nouveaux aliments sera ensuite abordée, ainsi que leur impact sur les liens sociaux et sur les questions de parenté entre Africains. Nous étudierons aussi le rôle de l'État dans la transformation des pratiques alimentaires, en mettant l'accent sur les périodes de difficulté en ville, par exemple lors de périodes de sécheresse et de disette. Enfin, dans une dernière partie, nous nous intéressons aux changements intervenus après la Seconde Guerre mondiale en retraçant la manière dont la consommation alimentaire des Africains répond alors à un nouveau contexte économique et social. Finalement, le présent article n'a pas seulement pour objectif de reconstituer l'histoire de ce que les familles africaines mangeaient à Bulawayo, mais aussi d'explorer une riche histoire de la survie en milieu urbain et des stratégies alternatives dans le contexte du projet colonial. 


\section{Carte $n^{\circ} 1$ et $2^{4}$}

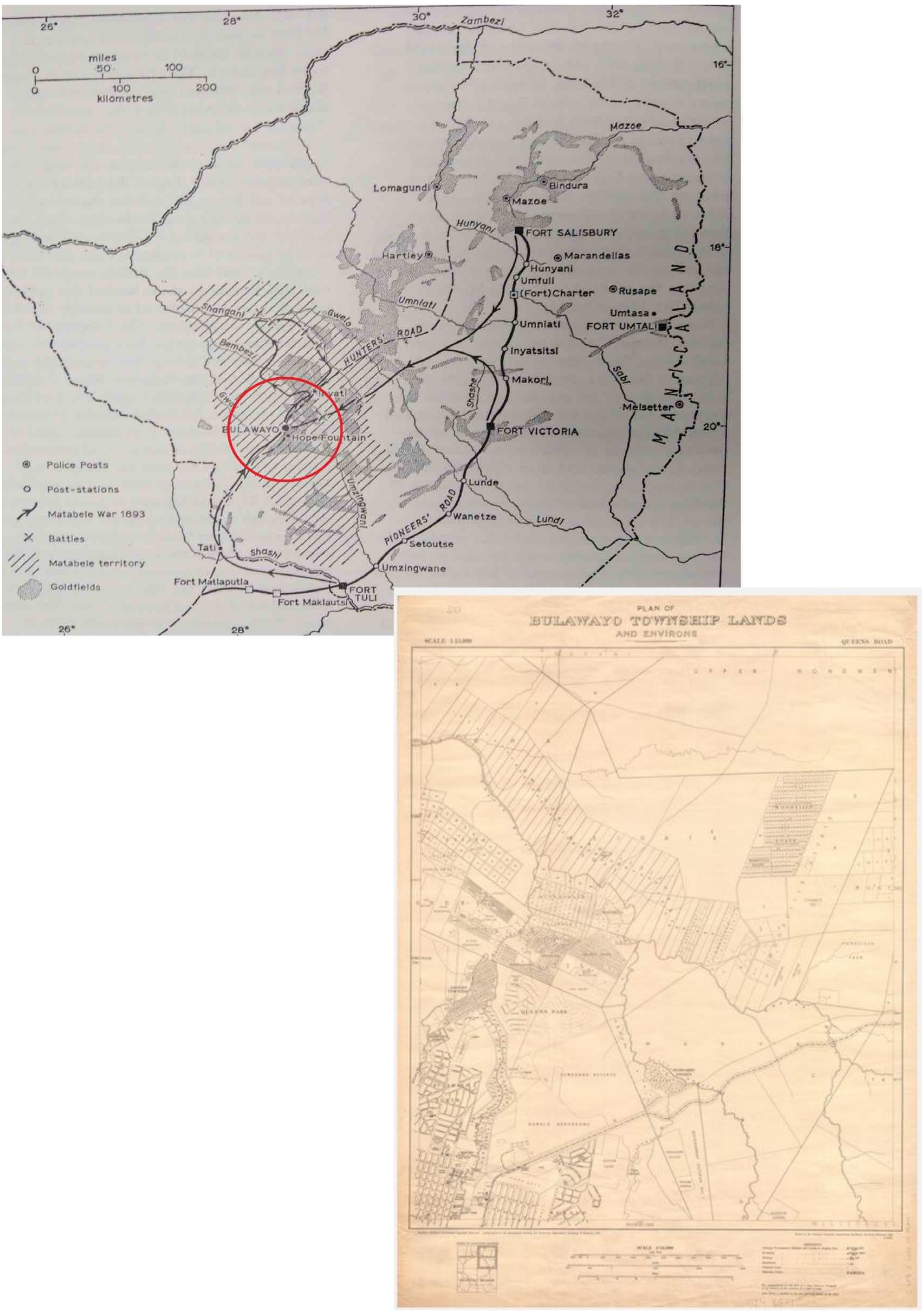

${ }^{4}$ Gale D.W. (1979), Rhodesia 1890-1970, Eighty Years onwards, Bulawayo, D.A. Blumberg, p. 23 ; Government of Southern Rhodesia (1950), Township Maps of Zimbabwe, Cape Town, University of Cape Town. 


\section{« Identités comestibles » : ville, alimentation et histoire}

Pendant de nombreuses années, l'histoire de Bulawayo coloniale et post-coloniale a été envisagée au prisme de la trajectoire historique du peuple ndebele, de son « ascension » à sa « chute $»^{5}$. Contrairement à Salisbury (actuelle Harare), Bulawayo a fait l'objet de peu de travaux scientifiques ${ }^{6}$. Des chercheurs tels que Tsuneo Yoshikuni' ${ }^{7}$ Teresa Barnes ou Everjoice Win ${ }^{8}$, dans leur ouvrage de 1992 intitulé To Live A Better Life, proposent une histoire sociale très complète des Africains dans les villes du Zimbabwe au début du XX⿳亠丷厂 siècle. Mais malgré leur richesse, ces études mettent en évidence une lacune historiographique à propos des agglomérations autres que Harare.

Pourtant, comme le rappelle Ennie Chipembere, Bulawayo est dans les années 1940 le centre économique de la colonie ${ }^{9}$ et dans son ouvrage intitulé Bulawayo Burning, Terence Ranger montre que la ville est, au début des années 1950, le berceau du nationalisme africain en Rhodésie ${ }^{10}$. À cette époque, les responsables des Native Affairs Department Annuals (NADA) présentent les Africains en mettant l'accent sur leur rôle de main-d'œuvre dans le développement du tabac ${ }^{11}$, du maïs ${ }^{12}$ et des produits laitiers ${ }^{13}$, mais ils négligent la riche histoire des communautés africaines en ville, en particulier à Bulawayo. L'historien Murray Steele analyse comment, dans le cadre de l'élaboration d'une politique coloniale du travail, les responsables de la British South African Company (BSAC) prennent conscience dans les années 1920 des dynamiques démographiques nouvelles qui mettent en évidence une présence africaine importante dans les villes, à la fois comme marché de main-d'œuvre et comme bassin de consommation pour les industries naissantes des colons ${ }^{14}$. Comme le soulignent des travaux tels que ceux de Fuller Masuku' ${ }^{15}$, la politique coloniale s'est largement efforcée de contrôler et

\footnotetext{
${ }^{5}$ Ranger Terence (2010), Bulawayo Burning: The social history of a Southern African City, 1893-1960, Harare, Weaver Press ; Musemwa Muchapara (2014), Water History and politics in Zimbabwe: Bulawayo's struggles with the environment, 1894-2008, Trenton, Africa World Press; Vera Yvonne (1998), Butterfly burning, Harare, Baobab ; Yoshikuni Tsuneo (2007), African Urban Experiences in Colonial Zimbabwe: A Social History of Harare before 1925, Oxford, African Books Collective.

${ }^{6}$ Soulignons toutefois les travaux fondamentaux des historiens Terence Ranger et Muchapara Musemwa, qui ont documenté l'histoire sociale de la ville et de ses habitants.

7 Yoshikuni T., African Urban Experiences..., op. cit., pp. 47-51.

8 Barnes Teresa et Win Everjoice (1992), To Live A Better Life: An Oral History of Women in the City of Harare, 1930-70, Harare, Baobab Books; Barnes Teresa (1999), "We Women Worked So Hard": Gender, Urbanization and Social Reproduction in Colonial Harare, Zimbabwe, 1930-1956, Portsmouth, N.H. Heinemann.

${ }^{9}$ Chipembere Ennie (2007), Colonial urban policy and Africans in urban areas, with special focus on housing, Salisbury, 1929-1964, Thèse de doctorat en histoire, Université du Zimbabwe, p. 112. Parmi les secteurs industriels et les entreprises notables qui avaient leur siège à Bulawayo pendant la période coloniale, citons David Whitehead Textiles, Gold Star (sucre), Cold Storage Company et Rhodesian Railways.

10 Ranger T., Bulawayo Burning..., op. cit., pp. 72-81.

${ }^{11}$ Ncube Sibanengi (2018), Colonial Zimbabwe Tobacco Industry: Global, regional and local relations, 1949-1979, Thèse de doctorat en histoire, Université du Free State ; Doro Elijah (2020), A socio-environmental history of commercial tobacco farming in Southern Rhodesia and Zimbabwe, 1893-2000, Thèse de doctorat en histoire, Université de Stellenbosch.

${ }^{12}$ Machingaidze Victor (1980), The development of settler capitalist agriculture in Southern Rhodesia with particular reference to the role of the state, 1908-1939, Thèse de doctorat en histoire, Université de Londres.

13 Hove Godfrey (2015), The State, Farmers and Dairy Farming in Colonial Zimbabwe (Southern Rhodesia), c.1890-1951, Thèse de doctorat en histoire, Université de Stellenbosch.

14 Steele Murray (1972), The foundations of native policy: Southern Rhodesia, 1923-1933, Thèse de doctorat en histoire, Université Simon Fraser.

${ }^{15}$ Masuku Fuller (1989), A study of agricultural change in the Ntabazinduna reserve with particular reference to the colonial period, 1923-1939, Mémoire de master, Université du Cap.
} 
réglementer le développement des Africains sur le plan agraire ${ }^{16}$. Cependant, des chercheurs comme Allison Shutt ${ }^{17}$ et Timothy Burke ${ }^{18}$ ont montré l'évolution de la politique coloniale, obligée de prendre en compte le besoin croissant, pour les citadins africains, de coller à l'image d'une « Rhodésie blanche ». Dans les années 1930, cette vision s'impose en ville vial'imposition de comportements raciaux (racial etiquette), d'une langue et d'une culture. Nous allons voir comment l'alimentation a suivi une trajectoire similaire.

En différentes occasions, comme lors des grandes sécheresses de 1922, 1942 et 1947, les modèles de consommation africains changent. Dans un contexte géographique très différent mais à la même époque, Kate Merkel-Hess a montré comment, durant la sécheresse de 1925, le gouvernement chinois introduit les réformes Dingxian (réformes alimentaires et agricoles) et remodèle ainsi les habitudes alimentaires pour qu'elles s'adaptent au « Mouvement de la Nouvelle Culture » (New Life Movement) ${ }^{19}$. En Rhodésie du Sud, les sécheresses récurrentes qui sévissent dans le pays à partir de la fin des années 1930 entraînent des pressions sociales et économiques croissantes qui conduisent les familles africaines à explorer des stratégies de survie alimentaire et de nouvelles façons de cuisine ${ }^{20}$. Eric Nobbs souligne qu'au cours de la première décennie du $\mathrm{XX}^{\mathrm{e}}$ siècle, les mines deviennent le débouché principal pour la production agricole des colons européens ${ }^{21}$ tandis que dans les zones rurales et urbaines, I'« assiette » africaine subit d'importantes transformations, qui reflètent la nature des cultures agricoles et les salaires des travailleurs noirs ${ }^{22}$. À partir de la fin des années 1940, les marchands ambulants de céréales et de viande se multiplient pour répondre à l'industrialisation rapide du pays. La ville est le témoin de changements dans la répartition des rôles des hommes et des femmes, à la fois dans la commercialisation et la préparation des aliments. Dans de nombreux périmètres industriels (coumpounds), de plus en plus d'hommes s'impliquent dans la cuisine et la restauration, tâches auparavant principalement effectuées par les femmes ${ }^{23}$. Les mutations complexes qui affectent la production alimentaire peuvent s'observer non seulement par-delà les barrières raciales, mais aussi par-delà les barrières sociales et dans les déplacements géographiques - de la campagne vers la ville, et vice versa. Après la promulgation de la loi sur les zones urbaines en 1943, la mobilité et la liberté des Africains deviennent particulièrement difficiles durant la décennie 1940. Cela a un impact sur leur alimentation traditionnelle dans les zones urbaines, mais aussi sur leur capacité à conserver leurs anciens usages: partager la

\footnotetext{
16 Ncube Trevor (1980), «Peasant production and marketing of grain crops in Zimbabwe, 1890-1980. An overview », Handerson Seminar Paper, 72.

17 Shutt Allison K. (2015), Manners Make a Nation: Racial Etiquette in Southern Rhodesia, 1910-1963, New York, University of Rochester Press.

18 Burke Timothy (1996), Lifebouy Men, Lux Women: Commodification, consumption and cleanliness in modern Zimbabwe, Durham, Duke University Press.

${ }^{19}$ Merket-Hess Kate (2016), The rural modern: Reconstructing the Self and State in Republican China, Chicago, University of Chicago Press, pp. 80-82.

20 Chigodora Joshua (1997), «Famine and Drought: The Question of Food Security in Zimbabwe », Drought Network News (19942001), p. 40 ; lliffe John (1990), Famine in Zimbabwe, 1890-1960, Gweru, Mambo Press, pp. 159-160.

21 Nobbs Eric (1906), " The mines as a market: The crops required for native diet », Rhodesian Agricultural Journal, p. 795.

22 Duggan William R. (1980), « The Native Land Husbandry Act of 1951 and the Rural African Middle Class of Southern Rhodesia », African Affairs, 79, pp. 227-239.

23 Samasuwo Nhamo (2003), «Food Production and War Supplies: Rhodesia's Beef Industry during the Second World War, 19391945 », Journal of Southern African Studies, 29(2), pp. 487-502.
} 
nourriture avec d'autres familles; en acheter sur les stands qui se multiplient et offrent des produits africains ${ }^{24}$.

\section{Politique et consommation alimentaires}

Des années 1920 aux années 1950, sous une pression sociale et économique grandissante, le régime alimentaire des Africains reflète le niveau des salaires souvent maigres des travailleurs, tout particulièrement dans les centres urbains. II ne fait aucun doute que cela a un impact sur le tissu social des familles africaines, avec un remodelage notable des rôles genrés et une multiplication des marchés ambulants, ainsi qu'avec le développement de l'aide alimentaire apportée aux foyers noirs, soutenue à la fois par l'État et les organisations philanthropiques. Comme nous le verrons plus loin, à partir des années 1930, le repas des Africains se transforme à mesure que de nouveaux besoins émergent en ville. Ces choix alimentaires et culinaires sont les reflets de la conjoncture économique, tandis que pour certains, ils deviennent des éléments essentiels de renforcement des liens sociaux.

À partir des années 1940, contrairement aux Africains des zones rurales, ceux qui vivent dans les townships urbains ne peuvent pas cultiver leur propre nourriture, du fait de la structure même des maisons ${ }^{25}$. Pendant un temps, cette conception des logements permet de maintenir les Noirs en position de purs consommateurs, les amenant à acheter davantage dans les magasins européens qu'à produire eux-mêmes leur nourriture ${ }^{26}$. Cependant, comme le montrent différentes études historiques, la consommation de petites céréales est considérablement limitée en ville. Pour preuve, Noel Vietmeyer, membre du Conseil national de la recherche de Washington, décrit à juste titre les petites céréales comme les «cultures perdues de l'Afrique ${ }^{27}$ », car leur consommation a pratiquement disparu. Dans I'historiographie, notamment dans l'ouvrage très complet intitulé The African Cookbook de Jessica Harris (2010) - qui traite des habitudes alimentaires, y compris en Rhodésie du Sud coloniale -, nul ne pourrait penser que les petites céréales ont fait partie du régime alimentaire des Africains, et encore moins prendre conscience de leur importance historique et sociale dans la vie des familles africaines ${ }^{28}$. Les habitudes alimentaires ont également évolué d'un point de vue géographique, en raison de l'évolution du peuplement des Africains en ville.

Dans certains cas, les modèles alimentaires, et l'usage des petites céréales en particulier, sont allés jusqu'à constituer une forme d'expression socioculturelle traduisant un besoin idéologique de la société africaine. Les historiens James McCannn ${ }^{29}$, Avital Livneh ${ }^{30}$ et Gerald

\footnotetext{
24 Phimister lan (1974), «Peasant Production and Underdevelopment in Southern Rhodesia, 1890-1914 », African Affairs, 73 , pp. 217228.

25 Jackson H.M.G., "Some reflections on the relation of Law to social anthropology », NADA, 1927, pp. 27-28.

26 West Michael O. (2002), The Rise of an African Middle Class: Colonial Zimbabwe, 1898-1965, Bloomington, Indiana University Press, pp. 55-69.

27 National Research Council (1996), Lost Crops of Africa: Grains, Washington, Board on Science and Technology for International Development, National Academies Press.

28 Harris Jessica (2010), The African cookbook: Tastes of a continent, New York, Simon and Schuster. Les petites céréales apparaissent moins de cinq fois dans l'ensemble des 382 pages. Ces dernières années, l'intérêt pour les petites céréales a grandi en grande partie à cause de préoccupations croissantes au sujet des sécheresses et des questions de sécurité alimentaire, partout dans le monde, et en particulier en Afrique.

${ }^{29}$ McCann James (2010), Stirring the pot: A history of African cuisine, Bloomsbury, Hurst and Co, p. 139.

30 Livneh Avital (1976), Precolonial polities in Southern Zambezia and their political communications », Thèse de doctorat, Université de Londres.
} 
Mazarire $^{31}$ soulignent que les petites céréales sont devenues de plus en plus importantes et ont été à la base de l'économie précoloniale des peuples shona et karanga au Zimbabwe. Une enquête ethnographique sur les Shona et les Ndebele, réalisée par l'anthropologue Hilda Kuper et ses collègues, montre qu'en 1910, le sorgho et le millet sont les principales cultures largement consommées à la fois par les Africains et les colons blancs, dans les premières années de la colonisation ${ }^{32}$. Selon I'historienne Thembani Dube $^{33}$, cette consommation généralisée de petites céréales par les familles africaines vaut à certaines des communautés kalanga du Zimbabwe le surnom de «peuple du millet». En nous inspirant de ces efforts antérieurs, nous souhaitons ici contribuer à l'étude les effets des changements dans les habitudes alimentaires sur la société africaine ${ }^{34}$.

\section{Le maïs : « L'ami rhodésien » ?}

Les produits agricoles africains dominent dans le cadre du marché céréalier qui se constitue dans les premières années de la Rhodésie du Sud. Africains et Blancs, tant ruraux qu'urbains, consomment de petites céréales et du maïs comme principales sources de glucides ${ }^{35}$. Avec l'expansion du secteur secondaire en ville - notamment les activités liées aux chemins de fer et à l'industrie textile -, la population africaine s'accroît à Bulawayo ${ }^{36}$. Logiquement, la demande de nourriture augmente également et, avant 1930, les marchands africains de céréales profitent largement de ce boom précoce. Montague Yudelman s'intéresse aux marchands de grains entreprenants qui s'installent à la périphérie des villes et des centres miniers pour vendre leurs céréales aux travailleurs des mines ${ }^{37}$. Cependant, en 1906, le directeur de l'Agriculture Eric Nobbs relaie les plaintes récurrentes des fermiers blancs qui déplorent que la Rhodésie ne développe qu'une production agricole, le maïs, et lance des enquêtes sur les possibilités de développer cette céréale de façon à ce qu'elle devienne un produit de base dans le pays, et qu'elle soit également exportée ${ }^{38}$. Ian Phimister a montré comment, en 1908, les colons blancs producteurs de maïs réussissent à faire pression sur la BSAC pour qu'elle promulgue I'Ordonnance sur I'Alimentation (Diet Ordinance), dont le but principal est de populariser le maïs cultivé par les Blancs afin de remplacer, auprès des ouvriers africains des mines et des industries, les petites céréales et le maïs produit et vendu par les agriculteurs et

\footnotetext{
31 Mazarire Gerald (2009), "Reflections on pre-colonial Zimbabwe, c.850-1880s », in B. Raftopoulos et A. Mlambo (dir.), Becoming Zimbabwe: A History from the pre-colonial period to 2008, Harare, Weaver Press, pp. 36-37. À côté de l'or, des semences et de I'ivoire, il s'agissait de produits-clés donnés en tribut aux autorités religieuses et politiques, de même qu'ils étaient utilisés comme produits d'échange dans le commerce avec les Portugais et avec d'autres sociétés africaines, le long de diverses routes commerciales. Voir également Wilmot Alexander (1895), The Story of the Expansion of Southern Africa, Londres, T. F. Unwin.

32 Kuper Hilda et al. (dir.) ([1954], 2017), The Shona and Ndebele of Southern Rhodesia, London, Routledge.

33 Dube Thembani (2015), Shifting identities and the transformation of the Kalanga people of Bulilimamangwe district, Matabeleland South, Zimbabwe, c. 1946-2005, Thèse de doctorat en histoire, Université du Witwatersrand.

34 Hastorf Christine A. (2017), The Social Archaeology of Food: Thinking about Eating from Prehistory to the Present, Cambridge, Cambridge University Press, p. 36.

35 Kauma Bryan (2021), " "Small grains, small gains": Small grains production and marketing in Southern Rhodesia ", South African Historical Journal, en ligne. URL : https://www.tandfonline.com/doi/abs/10.1080/02582473.2021.1938192 (consulté le 15 septembre 2021).

36 Nobbs E., « The mines as a market... », art. cité, pp. 795-796.

37 Yudelman Montague (1964), Africans on the land, Cambridge, Harvard University Press, pp. 174-175. Ce faisant, ils se rapprochent du marché, ce qui constitue une incitation supplémentaire à pratiquer le commerce des céréales dans les villes.

38 Nobbs E., « The mines as a market », art. cité, p. 795.
} 
les commerçants africains ${ }^{39}$. Bien que cette ordonnance ait un impact considérable sur I'alimentation des travailleurs noirs, les intérêts économiques parfois contradictoires des autorités blanches contribuent à sa mauvaise application. Dès lors, la consommation de petites céréales continue à prédominer parmi les familles africaines, par opposition au maïs plus coûteux produit par les colons blancs. En outre, ces céréales restent populaires en raison de leur utilisation sociale plus large, notamment pour le brassage de la bière artisanale (opaque beer) et pour la préparation de porridge et de sadza nutritifs dans les familles noires.

Jusque dans les années 1930, les travailleurs africains des mines et des chemins de fer constituent le principal marché de consommateurs pour les céréales en ville. Lors des repas du matin et du soir, maïs et petites céréales sont consommés surtout sous forme de sadza, en raison de leur richesse en glucides et en amidon, sources importantes d'énergie ${ }^{40}$. Au cours de la journée, une boisson non alcoolisée appelée maheu, produite également à partir de petites céréales, est consommée pour se rafraîchir et faire le plein d'énergie ${ }^{41}$. Lors de la préparation des repas, les petites céréales offrent une meilleure sensation gustative que le maïs. De plus, en 1922, le rapport du Commissaire en chef aux indigènes (Chief Native Commissioner, CNC) note qu'elles produisent une bière plus alcoolisée, appréciée des ouvriers et des Africains en général, et en particulier des hommes ${ }^{42}$. Pourtant, au début des années 1920, le directeur du Développement indigène (Director of Native Development), Emory Alvord, décrit ce qu'il appelle un " complexe du maïs », observant que « les indigènes eux-mêmes savent que le maïs est beaucoup plus facile et moins cher à cultiver que le rapoko et que son travail leur rapporte davantage $\mathrm{e}^{43}$ ». Pourtant, en raison d'une multitude de facteurs sociaux tels que leur utilité pour le brassage de la bière traditionnelle, les petites céréales sont l'objet d'une demande massive de la part des Africains ${ }^{44}$. Cette demande est renforcée par les mouvements migratoires de la main-d'œuvre africaine: initialement paysans ou petits producteurs de denrées agricoles destinées à la consommation du ménage, les travailleurs deviennent en ville des salariés. Ce phénomène entraîne une dépendance à l'égard des céréales achetées pour la consommation courante. La réglementation urbaine interdit aux Africains des villes de travailler la terre et les longues heures consacrées au travail industriel les privent de la possibilité, et des ressources nécessaires, pour cultiver leurs petites céréales préférées.

En 1922, Bulawayo subit une sécheresse qui touche toute la région du Matabeleland. Les pénuries alimentaires sont monnaie courante, aggravées par ce que le ministère des Affaires indigènes (Department of Native Affairs) décrit comme une mauvaise gestion des produits alimentaires par les ménages noirs ${ }^{45}$. Au cours des années 1920, l'État colonial ne réussit pas à s'attaquer de manière adéquate au problème de la faim, se préoccupant plutôt de réduire la

\footnotetext{
39 Phimister I., «Peasant Production... », art. cité, p. 220. Voir également Punt Eira (1979), The development of African agriculture in Southern Rhodesia with particular reference to the interwar years, Mémoire de master en histoire, Université de Natal, p. 74. Phimister et Punt soulignent que le maïs des Blancs est comparativement plus cher que celui produit par les Africains, et ce pour plusieurs raisons, notamment les contraintes liées au travail et à la terre qui limitent les rendements.

40 NADA, « Native food and culinary methods », 1933, pp. 101-104.

41 Ibid.

42 Archives Nationales du Zimbabwe (NAZ), 1095/06/S2, Southern Rhodesia, Report of the CNC, Insiza, 1922.

43 Madimu Tapiwa (2016), « Responsible Government and Miner-Farmer Relations in Southern Rhodesia, 1923-1945 », South African Historical Journal, 68(3), pp. 366-389.

44 Ibid.

45 NAZ, 1095/06/S2, Southern Rhodesia, Report of the CNC, 1923.
} 
production africaine de petites céréales et sa consommation par les mineurs et les citadins ${ }^{46}$. En ville, les difficultés économiques accrues, caractérisées par des salaires dérisoires et une déconnexion croissante par rapport à la vie familiale dans les réserves, amplifient les conséquences des sécheresses et des pénuries alimentaires chez les Noirs. Jusque dans les années 1930, ces vicissitudes déclenchent un besoin croissant de cultiver des petites céréales et du maïs chez les urbains ${ }^{47}$. En réponse, les autorités de Bulawayo imitent les initiatives des dirigeants locaux dans les districts miniers d'Insiza, Belingwe, Inyati et Umzingwani, en créant des caisses destinées à aider les Noirs: on leur fournit des céréales et d'autres produits alimentaires afin d'atténuer la disette et la crise de malnutrition qui menacent ${ }^{48}$. En 1947, le Comité national d'aide aux victimes de la sécheresse (National Drought Relief Committee), placé sous l'autorité du ministère de l'Agriculture et des Terres (Ministry of Agriculture and Lands), offre près de 102000 sacs de maïs et 27000 sacs de petites céréales à quelque 318000 Africains, pendant les quatre mois que dure la sécheresse qui sévit à Bulawayo ${ }^{49}$. Lors de cette distribution de nourriture, on fournit davantage de maïs que de petites céréales, produits pourtant plus prisés et mieux adaptés à l'environnement dans la région sèche du Matabeleland et de Bulawayo ${ }^{50}$. La persistance à vouloir nourrir les Noirs avec du maïs s'inscrit dans la campagne de "propagande et de persuasion douce » qui vise à protéger les intérêts des producteurs blancs en modifiant les habitudes alimentaires des Africains ${ }^{51}$. Cela renvoie en outre à ce que disait déjà en 1910 le directeur de l'agriculture de l'époque, Eric Nobbs: "Quelles que soient les autres cultures, le maïs doit rester la principale production agricole du pays $» .52$

Pourtant, malgré des réglementations anciennes comme l'Ordonnance sur l'alimentation de 1908, l'État colonial a du mal à contrôler la consommation des petites céréales africaines par les travailleurs noirs. Pour ce qui est des habitudes alimentaires, seuls ceux qui vivent très proches des Blancs, ou qui sont sensible à la notion de "blanchité », sont touchés par ces mesures de substitution des céréales, en raison de leurs contacts réguliers avec les autorités qui les mettent en place ${ }^{53}$. Des produits européens tels que le riz, les biscuits et les conserves de maïs, de lait et de haricots sont vendus dans des kiosques à destination des travailleurs urbains. Entre les années 1940 et 1950, les petites céréales perdent de leur popularité parmi un groupe social émergent, noyau de la bourgeoisie africaine en devenir. Les transformations socio-économiques liées aux modes de vie urbains de ce groupe l'incitent à choisir le maïs

\footnotetext{
46 Machingaidze V., The development of settler capitalist, op. cit., pp. 414-417.

47 Ibid., p. 425. Les Africains urbains dépendent fortement des céréales abordables produites dans les zones rurales.

48 Ibid.

49 NAZ, S968/90, National Drought Relief Committee: Food Control and Distribution, CNC, Circular No. 58, Food requirements and famine relief, 26 juillet 1947, p. 2.

50 Weinmann H. (1972), Agricultural research and development in Southern Africa, 1890-1923, Salisbury, Université de Rhodésie, pp. 79-83. Les tableaux montrent l'évolution de la production agricole et sa répartition selon les surfaces ensemencées en maïs et en petites céréales.

51 Alvord Emory D. (1948), «The progress of Native agriculture in Rhodesia », New Rhodesia, 15, pp. 1-32.

52 NAZ, 1095/01/S2, Report of the Director of Agriculture, Dr Nobbs, 1910.

53 Ncube T., «Peasant production... », art. cité, p. 7. Nous utilisons le concept de « blanchité » tel qu'il est théorisé par l'historien Rory Pilossof dans son livre de 2012, The Unbearable Whiteness of Being - pour désigner l'idéologie, les idées et, dans certains cas, la présence physique des colons blancs, considérés comme supérieurs sur le plan économique et social. La « blanchité » est au service des intérêts de l'État et des colons les plus puissants et, comme le montre cet article, à celui de quelques membres choisis parmi les élites africaines émergentes. Voir également Alcoff Linda Martin (2015), The Future of Whiteness, Malden, Polity Press, p. 11.
} 
comme denrée de base, tandis que les petites céréales ne sont utilisées qu'occasionnellement, en bouillie et pour les boissons alcoolisées.

II faut rappeler que société blanche et société africaine n'ont pas une vision homogène des changements alimentaires. Certains fonctionnaires coloniaux blancs sont ainsi de fervents partisans des petites céréales. Certes, en 1927, le secrétaire à l'Agriculture Charles Murray décrit à quel point son département est préoccupé par le régime alimentaire des indigènes, avant de déclarer avec arrogance que le régime alimentaire des Européens est de loin supérieur à celui des Noirs ${ }^{54}$. Une telle vision est nuancée en 1942 dans un document de la Commission Howman consacré à l'alimentation et au régime alimentaire des Africains ${ }^{55}$. Cette réévaluation va dans le sens de l'opinion qu'en a à l'époque le médecin Michael Gelfand (opinion qu'il réitèrera dans une publication postérieure ${ }^{56}$ ), selon laquelle les petites céréales fournissent en fait davantage d'éléments nutritifs que le maïs, tout en étant plus faciles et moins chères à produire ${ }^{57}$. Dans un ouvrage de 1971, Gelfand souligne qu'entre le milieu des années 1940 et la fin des années 1960, les femmes africaines ont eu raison de nourrir leur famille avec des petites céréales, car celles-ci fournissent un apport nutritionnel adéquat tout au long de la journée ${ }^{58}$. Dans la région aride de Bulawayo, le régime alimentaire africain repose sur le millet plutôt que sur le maïs, car il est mieux adapté au climat et aux sols arides ${ }^{59}$.

H. Weinmann rapporte que, jusqu'à la fin des années 1920, dans les domaines miniers et les fermes des colons, les céréales africaines, en particulier les petites céréales, atteignent un meilleur prix que le maïs sur le marché, que ce soit chez les Blancs ou chez les Noirs ${ }^{60}$. En fait, bien que les registres officiels ne le démontrent pas, le commerce informel des petites céréales atteste que la demande reste forte parmi les travailleurs des villes, tant pour leur valeur sociale que pour leur valeur nutritionnelle supérieure à celle du maïs produit par les Blancs ${ }^{61}$. Les Africains demeurent réticents vis-à-vis de la consommation du maïs vendu dans les magasins européens, le décrivant comme le résultat d'un « mélange de grains divers » peu appétissant, inadapté à l'exportation et dépourvu de valeur nutritionnelle ${ }^{62}$. Même si ces mélanges ne sont pas toujours détectables au moment de l'achat, lors de la cuisson et de la consommation, la texture et le goût mettent en évidence les manigances des commerçants ${ }^{63}$. II est en effet courant que des intermédiaires indiens et blancs s'adonnent à ces pratiques - combinant le sorgho avec du millet et du rapoko, et parfois même du maïs - pour répondre à la demande urbaine croissante en matière de céréales, ce qui en compromet la qualité64. Cela a un impact sur les choix alimentaires des travailleurs africains à partir des années 1930, en particulier dans les communautés à faibles revenus.

\footnotetext{
54 Weinmann H., Agricultural research, op.cit., pp. 59-60. C'est malheureusement l'hypothèse qui domine et qui contribue à la dépréciation de l'agriculture indigène.

55 Howman Roger (1943), "The native labourer and his food", South African Institute of Race Relations (SAIRR), p. 21.

56 Gelfand Michael (1971), Diet and Tradition in African culture, Édimbourg et Londres, Livingstone ed., pp. 45-46.

57 Ncube T., «Peasant production... », art. cité, p. 14

58 Gelfand M., Diet and tradition in African culture, op. cit., pp. 45-46.

59 Masuku F., « A Study of Agricultural... », art. cité, p. 19.

60 Weinmann H., Agricultural research..., op. cit.

61 Howman R., "The native labourer and his food... », art. cité, p. 22.

62 Jayne T. S. et Rubey Lawrence (1998), "Maize milling, market reform and urban food security: The case of Zimbabwe », World Development, 21(6), pp. 975-988.

63 NADA, « Native food... », art. cité, pp. 101-104.

64 Kauma B., " "Small grains, small gains" ... », art. cité, p. 27.
} 


\section{Les années de contrôle du maïs}

En 1930 est adoptée la Loi sur le contrôle du maïs (Maize Control Act). Ce qui, à l'origine, était conçu comme un mécanisme temporaire destiné à protéger les cultivateurs blancs contre les aléas de la crise économique mondiale ${ }^{65}$ devient un élément permanent de la politique agraire coloniale ${ }^{66}$. Cette loi s'avère également une arme pour combattre la concurrence africaine. Dans le même temps, par le biais d'une réglementation inégalitaire du marché de distribution des céréales, la Loi sur le contrôle du maïs est un instrument qui modifie les habitudes alimentaires des Africains, en permettant un accès faussé aux céréales ${ }^{67}$. Comme indiqué précédemment, en période de pénurie alimentaire, l'accès limité à ces denrées pousse de nombreuses familles africaines urbaines à s'en remettre à l'aide de l'État pour se nourrir, leurs choix alimentaires étant de plus en plus limités.

La Loi sur le contrôle du maïs définit la valeur des grains en fonction de l'endroit où ils sont vendus, soit en zone $A$, soit en zone $B^{68}$. Ces délimitations ont été créées afin de limiter I'accès des Africains aux zones commerciales lucratives réservées aux agriculteurs blancs. Cependant, quelques agriculteurs africains qui vivent sur des parcelles urbaines à la périphérie de la ville, comme à Warringham, Kensington et Ntabazinduna, peuvent pénétrer dans ces zones réservées aux Blancs. À mesure que les perspectives s'améliorent à la fin des années 1930, certains adoptent le "régime blanc », car ils en préfèrent les saveurs et la variété. Pour d'autres, il s'agit peut-être d'une occasion d'élargir leurs choix alimentaires, mais aussi d'afficher publiquement un nouveau pouvoir d'achat qui leur permet de se payer des aliments "blancs » plus coûteux ${ }^{69}$. De plus en plus souvent, le maïs devient la principale denrée consommée par les Noirs de la classe ouvrière urbaine, comme le souhaitaient les maïsiculteurs blancs ${ }^{70}$.

À la fin des années 1930, la ville devient une plaque tournante des « produits alimentaires blancs ", qui comprennent des marques chères de farine de maïs raffinée, du corned-beef, du lait condensé et du pain. Les petites céréales sont dès lors de moins en moins populaires et de plus en plus utilisées comme fourrage pour le bétail ${ }^{71}$. Au cours de différentes opérations de travaux publics et au gré de programmes de sensibilisation dans les townships, notamment à Pelandaba et Mzilikazi, des régimes diététiques qualifiés d'« européens » sont présentés aux familles africaines par des représentants des autorités et de certains groupes religieux et philanthropiques blancs comme une « bonne nourriture pour combattre la faim »-selon les mots du responsable de l'information du département des Affaires indigènes (Native Affairs Department), H. Preston ${ }^{72}$. Au milieu des années 1930, avec le succès de ces initiatives d'État, les conceptions culinaires des colons se répandent chez les Africains. Renforcée par leur participation croissante à diverses Églises occidentales, la consommation d'aliments « blancs »

\footnotetext{
65 Vickery Kenneth (1985), « Saving Settlers: Maize Control in Northern Rhodesia », Journal of Southern African Studies, 11(2), pp. 212234.

66 Machingaidze V., The development of settler capitalist, op. cit., pp. 414-417.

67 Masuku F., « A Study of Agricultural... », art. cité, p. 19.

68 Ncube T., «Peasant production... », art. cité, p. 11.

69 Ibid., p. 16.

70 Ibid.

71 Chigodora J., « Famine and drought... », art. cité, p. 40.

72 "Africans hunt for food as well as for muti », The Chronicle, 17 août 1958. Voir aussi "Food relief for Africans ", The Rhodesian Herald, 16 mai 1935.
} 
se développe et certains ménages africains expérimentent les pratiques des colons, en utilisant davantage de maïs que de petites céréales dans la préparation des repas. Au début des années 1940, la cuisine africaine se transforme, aidée en cela par l'adoption d'ustensiles, notamment de moulins à grains mécaniques. Adopter des recettes de style plus européen est un choix influencé par l'efficacité de la préparation des aliments, un aspect important pour les travailleurs contraints à de longues heures de travail. Pour le maïs, l'utilisation de broyeurs à moteur diesel se généralise, tandis que pour les petites céréales - et jusqu'à aujourd'hui, comme le montrent les figures 1 et 2 -, les Africains continuent à utiliser des mortiers et de pilons de bois ou de pierre ${ }^{73}$. Les mortiers de bois, contrairement aux broyeurs mécanisés, ne donnent pas du grain lisse. Et lorsque l'on utilise un mortier de pierre, il est courant que de petits granulés de minéraux et de terre se mélangent par erreur au grain moulu ${ }^{74}-$ ce qui en rend la consommation désagréable. Dans le même temps, certaines femmes africaines employées comme domestiques dans des foyers blancs acquièrent des compétences culinaires nouvelles, qui leur permettent d'expérimenter différentes textures de céréales ${ }^{75}$. Ces compétences, comme le fait remarquer Renata Coetzee, sont également mises en œuvre par les Africains lorsqu'ils cuisinent les petites céréales ${ }^{76}$. Pour les Africains qui adoptent ces innovations - notamment les conserves -, la préparation des aliments se trouve allégée et ils peuvent consacrer davantage de temps à d'autres activités ${ }^{77}$.

\footnotetext{
73 Weinmann H., Agricultural research..., op. cit., pp. 67-68.

${ }^{74}$ McCann J., Stirring the pot..., op. cit., p. 102.

75 Keigwin H. S., "Native development », NADA, 1956, pp. 10-11. Bien que la méthode traditionnelle du pilon en bois et de la pierre à moudre ait continué à dominer, les broyeurs mécanisés se sont répandus, offrant une nouvelle forme d'entreprise agricole à certains agriculteurs africains qui achetaient ces machines. Moyennant une petite rémunération, ils offraient des services de mouture. Voir Steen Karin (2011), «A time to farm: A qualitative inquiry into the dynamics of the gender regime of land and labour rights in subsistence farming, an example from the Chiweshe communal area, Zimbabwe », Lund Dissertations in Sustainable Science, 2, pp. 4551.

76 Coetzee Renata (1982), Funa and Food from Africa: Roots of traditional African food culture, Durban, Butterworths, p. 33.

77 Masenda A. (1987), The Food Production Committee and state food policy in colonial Zimbabwe during the 1940s, Mémoire de master, Université du Zimbabwe, p. 110.
} 
Figure $n^{\circ} 1$ : Cinginkosi Dube, une jeune femme ndebele, utilisant un mortier et un pilon de bois pour moudre du millet dans le township de Nkulumane, à Bulawayo, en décembre $2020^{78}$

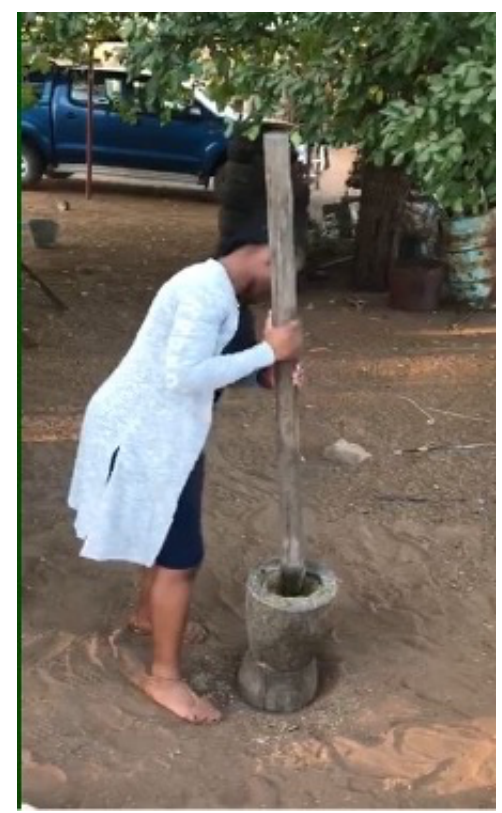

Figure $n^{\circ} 22$ : Famille africaine dans l'un des townships de Bulawayo, broyant du millet à l'aide de mortiers et de pilons de bois, au milieu des années $1990^{79}$

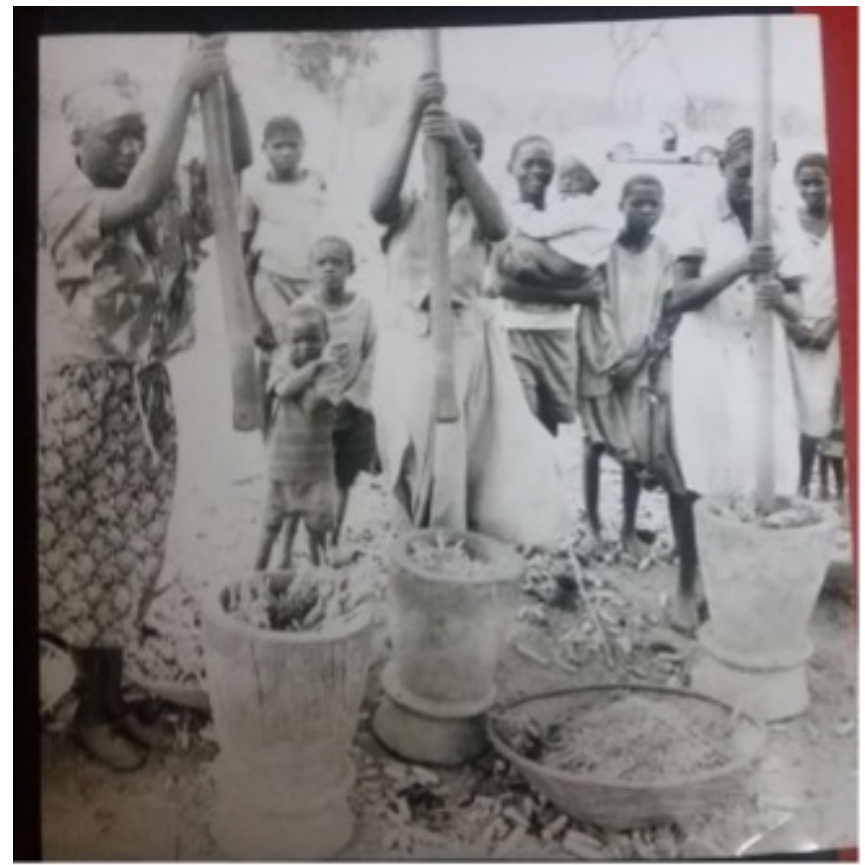

\footnotetext{
78 Image prise par Bryan Kauma dans le township de Nkulumane, à Bulawayo, en décembre 2020.

79 Image tirée de The Chronicle, File Box: Agriculture, iA8-IB2, Farmers Club.
} 


\section{Citrouilles, courges géantes et riz : la nouvelle alimentation africaine urbaine}

La Loi sur le contrôle du maïs facilite le développement de pratiques alimentaires et culinaires innovantes dans les régimes africains urbains ${ }^{80}$. Dans certains cas, ces changements reflètent une réalité socio-économique : le niveau dérisoire du salaire des Africains. Dans d'autres cas, les modèles culinaires procèdent de la rencontre entre ressources disponibles et politique de I'État. Dans le même temps, certains Rhodésiens noirs ainsi que certains travailleurs migrants africains (principalement venus de Rhodésie du Nord et du Nyassaland) conservent, même en ville, des habitudes culinaires et alimentaires traditionnelles, comme l'utilisation de pots en argile pour la cuisson et le séchage de la viande au soleil ${ }^{81}$. Bien qu'ils aient toujours recours à ces méthodes anciennes de cuisson, comme nous l'avons vu, la demande en petites céréales et en viande augmente jusqu'à la fin des années 1930, tant dans les périmètres miniers que dans les fermes des colons ${ }^{82}$. À Bulawayo, le régime alimentaire des Africains reste tributaire de l'approvisionnement en millet provenant des régions voisines, notamment de Ntabazinduna ${ }^{83}$. La proximité géographique entre les deux zones explique la disponibilité constante des petites céréales en ville, les agriculteurs des réserves s'étant lancés dans un commerce clandestin à destination des citadins afin d'augmenter leurs revenus. Dans les zones industrielles, différents magasins de produits alimentaires et d'épicerie accessibles aux Africains proposent une large gamme d'aliments, la plupart influencés par les goûts et les intérêts économiques européens, comme le riz et le pain ${ }^{84}$. Certains travailleurs africains sont progressivement initiés par leurs employeurs aux aliments "blancs » tels que riz, condiments épicés (même si, bien sûr, aucun d'entre eux n'est d'origine strictement européenne) et divers légumes. Entre 1939 et 1944, la production et la vente dans les compounds de produits cultivés par les Blancs - à savoir maïs, blé, orge, citrouilles et courges, tomates et légumes verts - augmente considérablement ${ }^{85}$. En 1943, un colon blanc fait remarquer que la cuisine africaine regorge de saveurs variées et que les menus font une place de plus en plus grande à quantité de légumes. Et avec le temps, ils deviennent esthétiquement attrayants et augmentent le plaisir gustatif des Africains, selon le responsable du Conseil de l'alimentation et de la nutrition, R. P. Denny, qui note que ces derniers « sont maintenant heureux quand ils mangent ${ }^{86}$ ».

Les changements apportés aux modes de préparation des petites céréales constituent une rupture avec ce que certains colons européens décrivaient comme une cuisine « monotone et malsaine ${ }^{87}$ ». Pour d'autres Blancs, la couleur brun chocolat ou rouge bœuf des préparations à base de petits grains qui emplissant les assiettes africaines provoque un sentiment de malaise sur le plan esthétique, également lié au choix des condiments (voir figure 3). Des commentaires

\footnotetext{
80 Guyer Jane (1987), Feeding African Cities: Studies in Regional Social History, Bloomington, Indiana University Press, pp. $46-48$.

81 Fairbridge Kingsley (1927), The autobiography of Kingsley Fairbridge, London, Oxford University Press, pp. 89-91.

82 Weinmann H., Agricultural research..., op. cit.

83 Masuku F., « A Study of Agricultural... », art. cité, p. 19.

84 Trevor N, «Peasant production and marketing... », art. cité, p. 7.

85 Annual agricultural reports from Rhodesian Agricultural Journal, 1937, 1939, 1940, 1944 and 1946 under the Heading Native Production in Southern Rhodesia.

86 NAZ, S482/781/39, Maize, Wheat, Grain etc, 1945-1960, communication between the Department of Native Affairs and the Secretary for Health, 15 janvier 1959.

87 R. M. Morris, Report on Public Health for the year 1949, Salisbury, Rhodesia Printing Company \& Young Farmers' Club pamphlet: Meals in a Shona home, 1969.
} 
comme celui-ci sont alors courants pour parler des menus africains à Bulawayo: « II semble y avoir une quantité considérable d'huile [... et] j'ose dire [... que] les Africains mangent les substances les plus indigestes comme condiments ${ }^{88}$. En 1928, la population noire de la ville monte à un peu plus de 12430 personnes, contre moins de 1200 Blancs, mais l'évolution rapide des aliments consommés suggère une influence croissante des seconds sur I'alimentation des premiers ${ }^{89}$. On constate que les kiosques locaux vendent aux travailleurs africains de plus en plus d'aliments " blancs », tels que du riz, des biscuits et des conserves ${ }^{90}$. Certains de ces produits ne sont pas hors de portée des travailleurs, qui sont de plus en plus conscients de la dimension sociale de cette nourriture. L'introduction de nouvelles denrées est aussi liée à des changements dans la manière dont les Africains cuisinent et mangent : dans de nombreuses cantines de la ville, il est désormais courant de prendre son repas en utilisant une fourchette et un couteau alors que, traditionnellement, les Africains aiment manger la sadza à la main ${ }^{91}$. Megan Vaughan explique que l'usage qui s'impose participe de la volonté de créer des « sujets gouvernables », en rendant acceptables certains aliments grâce la prescription de modes de cuisson et de consommation ${ }^{92}$.

Figure $n^{\circ} 3$ : Images de plats typiques de sadza (à base de petites céréales), consommés avec une variété de condiments traditionnels ${ }^{93}$

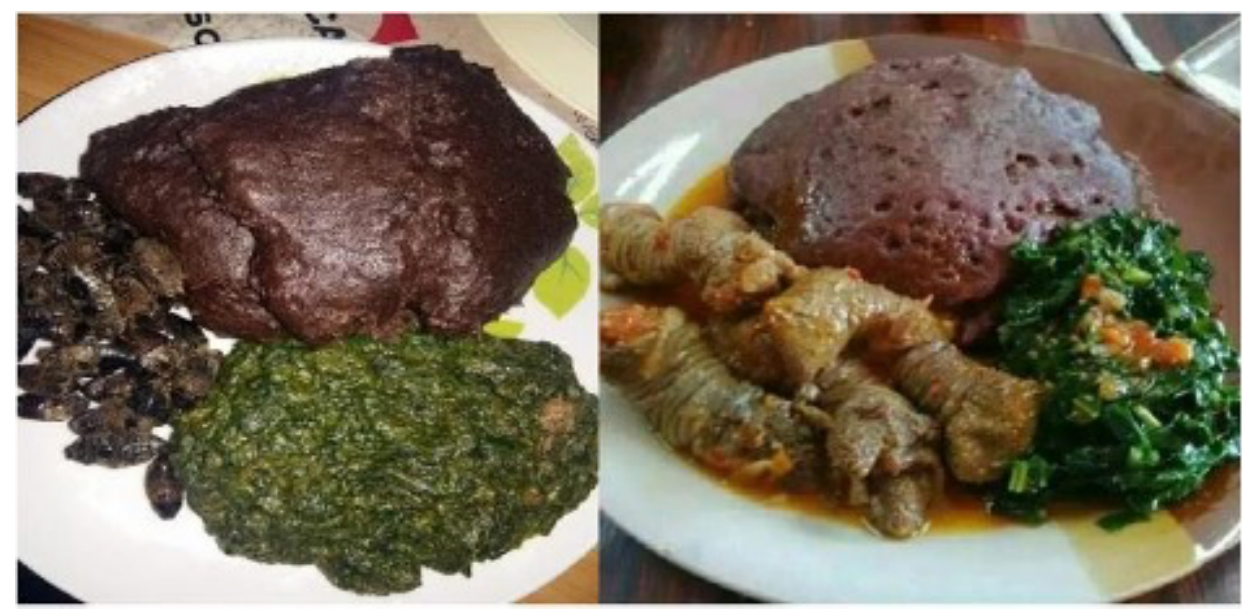

II ne fait aucun doute qu'une partie de ces méthodes s'avèrent trop compliquées pour certains ouvriers et ne contribuent que peu à l'« adoption » de certains aliments, comme le riz et le corned-beef ${ }^{4}$. En 1933, le CNC rapporte que les commerçants qui proposent des petites céréales et autres aliments traditionnels africains, tels que l'idelele (gombo) et l'amasi (lait aigre), font de très bonnes affaires en raison du faible intérêt des travailleurs noirs pour les aliments européens ${ }^{95}$. Pour d'autres, cette faible consommation est purement liée à des raisons

\footnotetext{
88 Meredith Martin (1979), The Past is Another Country: Rhodesia, Norfolk, Andre Deutsch, p. 67.

89 Secretary for Native Affairs: Report on the question of native housing and implementation of Land Apportionment Act in the Urban areas.

90 Howman R., "The native labourer and his food... », art. cité., pp. 32-44.

91 Haw R. G., "Some thoughts on Native development », NADA, 1950, pp. 20-23.

92 Vaughan Megan (1991), Curing their IIIs: Colonial Power and African IIIness, California, Stanford University Press, pp. $202-203$.

93 Images prises par Bryan Kauma lors d'une visite au Zimbabwe en décembre 2018.

94 Ibid., p. 24.

95 NADA, « Native food and culinary methods », 1933, p. 102.
} 
financières : comparés à la sadza de petites céréales, ces aliments nouveaux (riz, corned-beef et pain) atteignent des prix très élevés dans les magasins des complexes miniers et industriels ${ }^{96}$.

Comme l'affirme l'historienne Diana Jeater, à partir des années 1920, les approches et les instruments de la politique « indigène »-notamment dans les domaines de l'éducation et de l'alimentation - contribuent à la redéfinition des statuts sociaux, ce qui a un impact sur les modes de vie ${ }^{97}$. Par exemple, la consommation d'aliments comme que le riz contribue à stratifier la société noire en raison du statut que cette nourriture semble conférer, et de sa disponibilité dans certaines régions plutôt que d'autres ${ }^{98}$. En outre, l'introduction de légumes comme les citrouilles et les courges s'intensifie à partir des années 1930, ce qui signifie que la consommation de petites céréales est en perte de vitesse parmi l'élite africaine émergente, car elle est considérée comme une "culture du pauvre ${ }^{99}$ ». Disposant de davantage de moyens pour acquérir des ustensiles et des ingrédients de meilleure qualité et plus perfectionnés, les élites urbaines sont affectées par d'importants changements sociaux et économiques qui leur permettent d'intégrer à leur régime les aliments et les céréales contrôlés par le Maize Board (devenu Grain Marketing Board, GMB, en 1954). Ainsi, des denrées telles que le maïs, le riz et le blé deviennent leurs principales sources de glucides, tandis que les petites céréales ne sont plus utilisées qu'occasionnellement pour préparer le porridge ${ }^{100}$. Ce porridge du matin, préparé avec des petites céréales brunes épaisses, est peu à peu remplacé par du porridge préparé à partir de maïs, avec l'ajout facultatif de beurre et de sucre ${ }^{101}$. Auparavant, la plupart des femmes africaines ajoutaient des morceaux de citrouille et de canne à sucre pour adoucir la bouillie de petits grains et masquer sa saveur naturellement amère. Dans les deux cas, ces ajouts visaient à améliorer la saveur et à l'enrichir sur le plan nutritionnel.

Pathisa Nyathi rappelle que les Africains ont cultivé et consommé des aliments tels que les citrouilles depuis des siècles, mais que les principaux changements intervenus dans leur préparation ont consisté en l'ajout de divers condiments dont l'usage est à relier aux outils et aux ustensiles utilisés en ville ${ }^{102}$. Chez les Ndebele, certains aliments comme les farines, les citrouilles et les arachides ne sont pas consommés en repas complets, mais comme des « encas » tout au long la journée, en particulier durant les périodes d'activité intensive qui ne laissent pas suffisamment de temps pour préparer les repas ${ }^{103}$. En ville, la nourriture est elle aussi préparée la veille au soir et transportée dans un "skoftin » (boîte à casse-croûte) pour aller travailler. Le choix des produits consommés est également influencé par la possibilité de les consommer froids, car la plupart des travailleurs ne sont pas en mesure de les réchauffer. De plus, leur capacité à rester frais tout au long de la journée fait que ces aliments, dont le riz et les citrouilles, sont de plus en plus appréciés.

\footnotetext{
96 Masuku F., «A Study of Agricultural... », art. cité, p. 49.

97 Jeater Diana (2020), "African Women in Colonial Settler Towns in East and Southern Africa », Oxford Research Encyclopaedia, African History, Oxford, Oxford University Press, pp. 1-27.

98 Ibid., p. 12.

99 NAZ, 1905/F5, Abridged report of the Chief Native Commissioner in Belingwe for the Year ended 1935.

100 Mosley Paul (1983), The Settler economies: Studies in the economic history of Kenya and Southern Rhodesia 1900-1963, Cambridge, Cambridge University Press, pp. 40-43.

101 Howman R., « The native labourer ... », art. cité, p. 22.

102 Nyathi Pathisa (2020), African food, Bulawayo, Amagugu Publishers, p. 12.

103 lbid.
} 


\section{« Le goût de chez soi »}

Au début des années 1940, tant en ville que dans les réserves, la vie des Africains se transforme. La cuisine, plus que tout autre aspect social du foyer africain, subit une mutation radicale. Ces changements ne sont ni toujours imposés, ni toujours jugés indésirables : il s'agit plutôt d'un processus où se mêlent observation et appropriation. Des politiques nationales telles que la Loi sur les zones urbaines (Urban Areas Act) et la commission d'enquête Howman (Howman Commission of Inquiry) en 1943 officialisent par exemple des espaces où les populations noires et blanches peuvent interagir pour conduire des affaires et pour certains loisirs. L'Urban Areas Act réglemente également les espaces de production agricole autorisées dans les townships ${ }^{104}$. Les interactions économiques et sociales entre les communautés africaines et les colons se font plus courantes et plus étroites, dans la mesure où la main-d'œuvre africaine est de plus en plus nécessaire au développement des activités capitalistes blanches en ville. Les jardins familiaux africains dans les zones urbaines (et à un moindre degré dans les zones rurales) se multiplient et incluent une plus grande variété de légumes et d'herbes, qui sont utilisés pour enrichir la couleur, la saveur et la valeur nutritive des repas quotidiens. De ces interactions sociales plus fréquentes entre Noirs et Blancs, ainsi qu'entre les Noirs eux-mêmes, naissent des idées d'amélioration des maisons, qui sont adoptées par les femmes africaines, de plus en plus occupées et tiraillées entre travaux domestiques et petits emplois en complément des salaires de leurs maris ${ }^{105}$. Ainsi, les familles africaines modifient leurs habitudes alimentaires au fur et à mesure des changements socio-économiques importants qui surviennent dans le pays. Cependant, en adoptant certaines nouvelles conceptions culinaires et alimentaires, tous les Noirs ne négligent pas leur consommation de petites céréales. Au contraire, certains continuent à les inclure dans leurs repas désormais agrémentés d'une variété de légumes ${ }^{106}$.

Au fur et à mesure que la coexistence des Noirs et des Blancs va grandissant, la société urbaine africaine se stratifie en classes sociales diverses, ce qui a des conséquences sur la façon dont on cuisine et mange. Les visites régulières « à la maison » (dans les réserves « indigènes ») permettent aux Africains de partager de nouveaux aliments, ingrédients et conceptions alimentaires propres à la ville, mais elles donnent aussi libre cours à la nostalgie, leur donnant l'occasion d'apprécier certaines des petites céréales qui se font plus rares en milieu urbain. Lorsqu'ils reviennent en ville, ils emportent avec eux des sachets de petites céréales que certains partagent ou échangent, ce qui permet aux familles citadines éloignées de leurs foyers de rester en contact avec leurs racines ${ }^{107}$. Mais dès 1942, malgré ce lien aux aliments des réserves indigènes, qui permettent de maintenir les relations de parenté, de plus en plus d'urbains privilégient désormais la nourriture «blanche »108. En 1956, évoquant la disparition

\footnotetext{
104 La loi sur les zones urbaines (Urban Areas Act) définit les zones où Noirs et Blancs peuvent se rencontrer, en délimitant des espaces résidentiels, économiques et industriels (travail) et de loisirs. Un résultat notable de cette loi est de limiter la consommation de boissons traditionnelles alcoolisées (à base de petites céréales) aux tavernes surveillées par la municipalité et situées dans les townships.

105 NADA, « Native food and culinary methods », 1933, p. 101.

106 NAZ, S482/781/39, Maize, Wheat, Grain etc, 1945-1960, communication between the Department of Native Affairs and the Secretary for Health, 15 janvier 1959.

107 Williams-Forson Psyche (2014), « "I Haven't Eaten If I Don't Have My Soup and Fufu": Cultural Preservation through Food and Foodways among Ghanaian Migrants in the United States », Africa Today, 61, pp. 68-87.

108 Kuper H. et al, The Shona..., op. cit., pp. 24-28.
} 
de la consommation de sadza109, un rapport note que désormais, en ville, "les Africains sophistiqués [...] ne mangent pas de ces plats-là ${ }^{110} »$. Cette remarque renvoie au point de vue de William Whit sur la relation asymétrique entre zones rurales et urbaines, qui se manifeste de diverses manières et y compris sur le plan culinaire ${ }^{111}$. À Tjotsholo, certains travailleurs africains sont connus pour se pavaner dans le village en bleu de travail lorsqu'ils rendent visite à leurs familles. Beaucoup apportent de la ville des provisions et un indicateur important de leur présence est la cuisson de vetkoek (ou «fatcakes»), sortes de brioches confectionnées à partir d'un mélange de farine de blé et de poudre levante, et dont l'arôme flotte dans l'air ${ }^{112}$. Pour les familles rurales, l'équivalent de ces appétissants petits pains est l'amaqebelengwane, fait à partir de petites céréales mélangées à de la farine de maïs, mais cette préparation ne dégage pas l'arôme parfumé des vetkoeks. Dans les années 1950, la consommation de "fatcakes » est devenue un indicateur de l'acceptation sociale d'un individu à l'égard du style de vie dit moderne. À cette époque en effet, au sein du village - et surtout pendant les périodes de sécheresse -, l'accès à la ville est une composante tangible du statut social des individus ${ }^{113}$. En termes socio-économiques simples, « les gros chats mangent des gâteaux gras ».

Notons que le nombre d'hommes africains employés et résidant dans les townships urbains passe de 15322 en 1936 à environ 48000 en 1946114. Parallèlement, à partir du milieu des années 1940, de nombreuses femmes africaines viennent résider en ville. Celles qui émigrent à Bulawayo occupent des espaces dans les zones à forte densité dominées par les hommes célibataires : en 1945, elles ont ainsi investi une partie du marché alimentaire et établi de nouveaux commerces. Les repas prisés par les ouvriers africains comprennent des plats à base de petites céréales et de la bière traditionnelle. Dans l'ère postérieure à la Loi sur le contrôle du maïs, les petites céréales, autrefois très populaires, sont moins fréquentes ${ }^{115}$, mais les travailleurs africains, fatigués des régimes limités et monotones fournis par les mines, préfèrent les repas à base de ces céréales traditionnelles, et sont prêts à payer parfois beaucoup plus cher pour les obtenir ${ }^{116}$. Pour les commerçantes, cela " paye mieux et [c'est] une vie plus agréable ", ce qui témoigne d'une conscience de l'évolution du marché alimentaire ${ }^{117}$. Ces commerces se maintiennent car les stands de nourriture africaine sont également des lieux propices à la nostalgie, où les hommes éloignés de chez eux peuvent rester en contact avec leurs origines ${ }^{118}$. Ainsi, ces étals ne servent pas seulement à acheminer des produits africains introuvables dans les magasins, mais constituent aussi des nœuds de communication reliant

\footnotetext{
109 «African culture has changed», The Rhodesian Chronicle, 15 May 1952.

110 Keigwin H. S., "Native development », NADA, 1956, pp. 10-13. Voir aussi Kayongo-Male Diane et Onyango Philista (1984), The sociology of the African family, London, Longman, pp. 34-36.

111 Whit William (1999), "Soul Food as Cultural Creation », Journal for the Study of Food and Society, 3(1), pp. 37-47.

112 Desmond Judy (1963), Traditional Cookery in Southern Africa, Cape Town, Books of Africa, pp. 12-14. Desmond note que dans d'autres régions d'Afrique australe, des petits pains similaires cuits à la farine sont appelés vetkoek en afrikaans ou amagwinya en siXhosa et en isizulu.

113 Interview avec Gogo Thokozani Nyathi, à Maphisa (Matobo District), 14 décembre 2019.

114 Ashton H. (1957), African administration in Bulawayo, (Bulawayo), pp. 3-5 cité par Preben Kaarsholm (1995), « Si Ye Pambili-Which Way Forward?: Urban Development, Culture and Politics in Bulawayo », Journal of Southern African Studies, 21(2), pp. $225-245$.

115 Phimister I., « Peasant Production... », art. cité, p. 219.

116 NAZ, F1071/11, Report of the Secretary for Native Affairs, General statistics on African production and native life, 1951.

117 Phimister I., "Peasant Production... ", art. cité, p. 218. Et ce, malgré les coûts de production de la farine, du transport et de la sécurité de ce commerce clandestin, qui font que ces étals mobiles ne peuvent pas fonctionner aussi souvent que la demande l'exigerait.

118 Williams-Forson P., «"I Haven't Eaten If... », art. cité.
} 
les familles rurales à ceux qui travaillent dans les compounds ${ }^{19}$. Le goût convoque la mémoire : ces repas sont importants, peut-être en raison de la saveur unique des plats à base de petites céréales, cuisinés selon les souvenirs de la vie antérieure des hommes ${ }^{120} \ldots$ C'est « le goût de chez soi ${ }^{121} »$.

S'ajoutant aux opinions des Blancs sur les régimes alimentaires africains, et en dépit de vues parfois dissonantes comme on l'a vu, de nouvelles préoccupations se font jour: elles se concentrent sur la valeur nutritionnelle des aliments, sur l'afflux de femmes en ville et sur le commerce de plats à base de petites céréales qu'elles pratiquent. En 1941, une nutritionniste blanche (qui, en 1962, sera nommée dans le district nord du Matabeleland par le ministère de l'Agriculture pour s'occuper de l'éducation culinaire des paysans) observe que le ministère des Affaires indigènes (Native Affairs Department) cherche à améliorer le régime des Africains en augmentant la variété des "bons aliments", tels que la farine de maïs, le pain et les légumes disponibles à la vente dans les magasins destinés aux citadins noirs ${ }^{122}$. En conséquence, certains employeurs, notamment dans le secteur du textile, offrent à leurs employés des colis alimentaires comprenant de la farine de maïs et de blé, et du riz ${ }^{123}$. Cela contribue encore à attiser le goût des Africains pour les aliments « blancs »124.

\section{Faim, paternalisme d'État et alimentation africaine}

Les périodes de sécheresse et de pénurie alimentaire donnent lieu à des initiatives d'aide alimentaire de la part de l'État et d'intervenants tels que les Églises, qui contribuent aussi à déterminer ce que les Africains de la ville mangent, et comment ils doivent le faire. Historiquement, l'un des avantages des familles rurales par rapport à leurs homologues urbaines est la possibilité d'avoir accès à des alternatives alimentaires, en particulier pendant les saisons de faibles récoltes, comme lors de sécheresses ou d'épidémies. En ville, les efforts de la municipalité pour lutter contre la faim entraînent des changements matériels et idéologiques dans la façon dont les Noirs préparent et consomment leur nourriture. La création d'un centre de distribution de nourriture à Bulawayo, géré par la Southern Rhodesia Missionary Conference, marque par exemple un tournant dans la programmation et l'élaboration des politiques alimentaires du pays, en raison des idées diététiques qu'il diffuse dans le cadre de la lutte contre la faim, entre 1903 et $1945^{125}$. Au début, les repas proposés se composent principalement de petites céréales, mais elles sont progressivement remplacées par du maïs, à mesure que les colons blancs gagnent de l'influence politique dans le pays et que le maïs devient plus populaire parmi les citadins ${ }^{126}$. Lors de la sécheresse de 1947 , les repas servis aux

\footnotetext{
119 Ncube T., «Peasant production... », art. cité, p. 13

120 NADA, « Native food and culinary methods », 1933.

121 Cusack Igor (2000), "African Cuisines: Recipes for Nation-Building? », Journal of African Cultural Studies, $13(2)$, p. 207.

122 Allaart L. K. (1982), «Education for nutrition », The Central African Journal of Medicine, 28(8), pp. $195-197$.

123 Masenda A., The Food Production Committee..., op. cit., p. 65.

124 NAZ, 1095/08, Report of the Secretary for Native Affairs to Director of Native Development, 1949.

125 Ruzivo Munetsi (2017), « Ecumenical initiatives in Southern Rhodesia: A history of the Southern Rhodesia Missionary Conference, 1903-1945 ", Studia Historiae Ecclesiaaticae, 43(1), pp. 1-17. Le tout premier centre de distribution alimentaire soutenu par le gouvernement et l'Église a été mis en place à Bulawayo en 1896. Pendant plus de sept ans, le centre sert une moyenne de 3000 personnes par jour.

126 Yoshikuni T., African Urban Experiences..., op. cit., pp. 46-49. En outre, les régimes alimentaires étaient conformes aux conceptions religieuses des Blancs concernant les aliments considérés comme impurs et tabous. Cela ne correspond pas toujours à une perception similaire et favorable parmi les populations africaines.
} 
Noirs sont composés de " céréales indigènes et de farines fournies à raison de 2 livres par tête et par jour, ou 1 livre et demi de riz ». Dans le même temps, une plus grande variété de légumes, de pommes de terre et de haricots, produits par les fermiers blancs, deviennent des choix alimentaires appréciés.

Les inquiétudes des Blancs portent sur la persistance de nombreuses pratiques considérées comme non hygiéniques et sur la consommation importante de bière traditionnelle par les consommateurs de petites céréales ${ }^{127}$. On «injecte » sur les marchés locaux des aliments " blancs ", comme de la farine de blé pour le pain, du riz, des pommes de terre et du lait concentré. Même après que se sont envolées les craintes de famine, l'État maintient des points de distribution d'aide alimentaire, transformant les townships en espaces où une large variété d'aliments est facilement accessible ${ }^{128}$. Lors des périodes de sécheresse et de disette, il n'est pas rare que les Africains se tournent vers des aliments auparavant évités, comme la viande de chien, de grenouille ou de souris - viandes socialement raillées par les Ndebele en raison de leur caractère peu hygiénique - et certaines variétés de petites céréales ${ }^{129}$. Cependant, ils ne laissent pas toujours la faim dicter leurs pratiques alimentaires. Pour soulager la sécheresse lors des grandes disettes de 1932 et 1947, I'État colonial distribue par exemple aux familles africaines urbaines des repas, très impopulaires, à base de maïs jaune et de casava $^{130}$. S'adressant au Conseil municipal le 12 octobre 1949, M. Dazinger note que ces céréales jaunes sont boudées par de nombreux Noirs, principalement en raison de leur utilisation historique comme fourrage destiné au bétail sur les domaines agricoles des colons $^{131}$. De plus, ce grain jaune, comparé aux autres repas consommés, a un goût désagréable et dégage un arôme fétide à la cuisson ${ }^{132}$. À cette époque, à la différence du maïs blanc ou des petites céréales, les populations africaines des villes ne l'apprécient pas du tout, eux dont les assiettes s'emplissent d'aliments de plus en plus variés et appétissants. Et naturellement, les repas à base de kenya ${ }^{133}$ - comme on appelle ce grain jaune - sont associés de façon négative à la sécheresse, à l'amertume, à la souffrance et à la pauvreté. Malgré la faim, cette variété de maïs est donc mal accueillie par les citadins africains ; à cause de ce rejet, il devient courant, dans de nombreuses familles, de rechercher de nouveaux accès à la nourriture.

Tandis que certains hommes travaillent par exemple dans les mines et les industries, d'autres travailleurs, notamment des femmes, sont employés comme aides domestiques ou jardiniers dans des foyers blancs, les repas sur place étant intégrés à leur salaire ${ }^{134}$. John Iliffe observe comment ces interactions croissantes entre familles africaines urbaines et Blancs au cours des années 1940 ont rapproché les représentations des différents aliments en comblant un fossé social, et il souligne la capacité à exercer des choix culinaires malgré la faim qui menace. Les africains sont conscients des mutations alimentaires qui se produisent autour

\footnotetext{
127 Moyo P. H., « Native life in the reserves », NADA, 1925, pp. 47-55.

128 Ibid.

129 NAZ, N3/11/7, Drought and Famine report, Native Department, 1952. Chez les Ndebele, le rapoko était parfois considéré comme désagréable, notamment en raison de son goût plus amer parmi les variétés à petits grains.

130 NAZ, 1095/-6/S2, Southern Rhodesia drought relief, CNC report, 1947.

131 «An African problem », The Chronicle, 15 octobre 1949. Also see Weinmann H., Agricultural development..., op. cit.

132 Ibid.

133 Aujourd'hui, cette farine de maïs est appelée « kenya », un terme quelque peu péjoratif dont l'origine reste incertaine, mais qui remonte à l'après-guerre et semble faire référence aux « étrangers ».

134 Iliffe John (1987), The African Poor: A History, Cambridge, Cambridge University Press, pp. 153-155.
} 
d'eux. Dans les chemins de fer, par exemple, les travailleurs expriment leur mécontentement lors du passage des petites céréales au maïs, céréale qui, de surcroît, est servie en plus petites portions que celles auxquelles ils sont habitués ${ }^{135}$. Si les plaintes des Africains concernant la nature de la nourriture sont monnaie courante, les protestations des travailleurs noirs consistent principalement, au milieu des années 1940, à exiger que les aliments soient mieux cuisinés et que les portions soient plus importantes ${ }^{136}$. Mais contrairement au cas du kenya, ils réagissent positivement aux repas servis dans les entreprises industrielles. À la fin des années 1940, le comité consultatif sur l'alimentation (Food Advisory Committee) au sein du ministère de l'Agriculture « découvre » que les plaintes concernant la nourriture sont dues au fait que le personnel chargé de distribuer les rations alimentaires aux travailleurs possède peu de connaissances en matière de nutrition ${ }^{137}$. Le résultat est la mise en place d'un nouveau régime alimentaire préconisé pour les ouvriers (voir tableau 1 ).

Tableau $n^{\circ}$ 1: Régime alimentaire africain préconisé et prix d'achat ${ }^{138}$

\begin{tabular}{|c|c|}
\hline Aliment & Prix \\
\hline Maïs & 4 pence la livre ou $1,30 £$ la tonne \\
\hline Riz & Entre $51 / 2$ et 6 pence la livre \\
\hline Viande (bœuf) & Entre $21 / 2$ t 3 pence la livre \\
\hline Citrouilles & Entre $1 / 5$ et $1 / 4$ de pence la livre, ou entre 30 et 40 shillings la tonne \\
\hline Blé & $2,30 £$ la livre \\
\hline Patates douces & 1 penny la livre, ou $8 f$ la tonne \\
\hline Pommes de terre & $1 \frac{1}{2}$ penny la livre, ou $12 £$ et 10 shillings la tonne \\
\hline Oignons & 2 pence la livre, ou $16 £$ la tonne \\
\hline Haricots & Entre 2 et 3 pence la livre, ou entre $16 £$ et $24 £$ la tonne \\
\hline Lait & Entre $1 \frac{1 / 2}{2}$ t $2 \frac{1}{2}$ pence les 50 litres \\
\hline Arachides & Entre $1 \frac{1 / 2}{2}$ et 2 pence la livre, ou entre $12 £$ et 10 shillings et $16 f$ la tonne \\
\hline
\end{tabular}

La municipalité de Bulawayo intervient pour mettre en œuvre des « mesures contre la famine » afin de s'assurer que les industries locales ne sombrent pas en raison de la migration vers d'autres régions d'une main-d'œuvre en quête de nourriture ${ }^{139}$. Toutefois, en raison de contradictions et de conflits internes, elle autorise rapidement les Africains à poursuivre les modes de production et de consommation alimentaires dits "traditionnels", tant que cela permet de lutter contre le fléau de la faim en milieu urbain ${ }^{140}$. En conséquence, les Africains recourent de plus en plus (de façon désormais invisible) à des formes de céréaliculture urbaine

\footnotetext{
135 Nehwati Francis (1970), "The social and communal background to "Zhii": The African riots in Bulawayo, Southern Rhodesia in 1960 », African Affairs, 69, pp. 250-266.

136 Ibid., p. 253.

137 Maravanyika Simion (2012), "Local responses to colonial evictions, conservation and commodity policies among the Shangwe », African Nbula, 5, pp. 1-20.

138 Ibid.

139 Kuper H. et al, The Shona..., op. cit., pp. 24-28.

140 Ibid.
} 
dans leurs arrière-cours ou dans des bosquets proches de leurs habitations ${ }^{141}$. La culture domestique du maïs prolifère dans toute la ville, en raison de l'intérêt grandissant pour cette céréale et de ses faibles exigences en superficie et en main-d'œuvre par rapport aux petites céréales et aux légumes. Ce phénomène suscite une nouvelle série de défis pour l'État colonial : freiner les migrations illégales des Africains; contrôler les commerces informels de denrées alimentaires; limiter la culture sur les berges des cours d'eau, car elle nuit à l'approvisionnement en eau de la ville ${ }^{142}$. Le coût de la vie pour un citadin augmente de $100 \%$ entre 1939 et 1947 ; dans ces conditions, les Africains incapables de produire leur propre nourriture souffrent de la faim. Pour faire face au coût élevé du maïs blanc, certaines familles varient leurs menus et leur choix de glucides en alternant entre maïs, petites céréales, riz et pain ${ }^{143}$. Lorsque l'on consomme des petites céréales, il devient courant de les accompagner de toute une variété de nouveaux ingrédients et de condiments pour atténuer leur goût prononcé et amer ${ }^{144}$. Il émerge ainsi une culture culinaire hybride, fusion de la «tradition » et de la " modernité », fruit des aléas économiques et des mutations du statut social, mélange de choix et de nécessité.

\section{«Masiye-phambili45 »? Changements et continuités dans les cuisines africaines}

Dans l'après-guerre, on continue à observer des changements au sein des foyers africains, sous l'influence des campagnes menées par l'État et par certaines organisations philanthropiques et visant à modifier les pratiques alimentaires des Africains, en fonction de la conjoncture économique et dans l'intérêt des colons blancs ${ }^{146}$. À partir des années 1950 , les jardins familiaux et communautaires d'inspiration blanche, qui permettent de cultiver de nombreux légumes et herbes aromatiques dans les townships, sont désormais monnaie courante, ce qui signifie que les citadins noirs adoptent les idées nouvelles ${ }^{147}$. Ce développement des cultures vivrières est soutenu par d'importantes campagnes de formation culinaire menées par l'État, comme l'«École sur roues » (School on Wheels) qui s'intéresse à la préparation des aliments avec pour objectif d'améliorer le régime alimentaire des urbains ${ }^{148}$. En 1942, I'initiative "School on Wheels » consiste essentiellement en un programme d'économie domestique et d'éducation culinaire et nutritionnelle, conçu par le Native Food and Diet Committee au sein du ministère des Affaires autochtones (Native Department) ; elle est destinée aux familles africaines des différents townships de Bulawayo ${ }^{149}$. Les leçons comprennent la présentation de différentes

\footnotetext{
141 Ncube T., « Peasant production... », art. cité, p. 14.

142 Ranger T., Bulawayo burning..., op. cit., pp. 150-156.

143 Nyathi P., African foods..., op. cit., p .21.

144 NAZ, G1/6/4/67, Annual Reports on Native Development 1936-1942.

145 Masiye phambili signifie «Allons de l'avant ». Cette expression est devenue la devise de la ville de Bulawayo et elle figure sur ses armoiries.

146 Page Sam L et Page Helena (1991), « Western hegemony over African agriculture in southern Rhodesia and its continuing threat to food security in independent Zimbabwe », Agriculture and Human values, 8, p. 11.

147 NAZ, 1090/F5, Munga and kaffir corn, Report of the Secretary of Native Affairs, CNC and Director of Development, 1956.

148 Masenda A., The Food Production Committee..., op. cit., p. 110-112.

149 NAZ, 1095/08, Report of the Secretary for Native Affairs, Chief Native Commissioner and Director of Native Development, 1949. En février 1942, la première session de la "School on Wheels », qui a lieu dans le township de Makokoba, attire 31 femmes africaines sur les 110 prévues au départ. Cette faible participation peut être attribuée à la dureté des conditions de vie dans les villes des années 1940, qui se caractérise par un nombre inférieur de femmes et d'enfants par rapport aux hommes. En 1956, sur un public
} 
variétés de maïs comme le Rhodesian Hickory King, le Salisbury White et l'Eureka Field. On explique comment les cuisiner et les consommer, et comment se nourrir de façon adéquate ${ }^{150}$. Les élèves apprennent à préparer des repas simples, mais de bonne valeur nutritionnelle. Les leçons se concentrent sur les méthodes de cuisson et promeuvent l'utilisation des poêles à charbon plutôt que du bois de chauffe couramment utilisé151. En dépit d'un faible taux de participation, la découverte de nouveaux ingrédients et l'apprentissage de méthodes de cuisson semblables à celles qui existent dans les foyers blancs se diffusent en ville.

Peut-être certaines conceptions de la cuisine enseignées renforcent-elles les préjugés à l'égard des modes de vie "traditionnels » des Noirs ${ }^{152}$. Des efforts sont déployés pour réguler le flux des Africains et de leurs produits alimentaires ${ }^{153}$, et une conséquence visible de cette politique est le développement de mesures de protection contre la « contamination africaine » et contre les mauvaises habitudes d'hygiène; tout cela se traduit par la volonté paternaliste d'enseigner des manières correctes de cuisiner et de manger ${ }^{154}$.

Comme nous l'avons vu, dès les années 1940, les régimes et les choix alimentaires des Africains se sont considérablement élargis en raison des contacts plus étroits avec les Blancs et avec l'alimentation qu'ils privilégient. L'amélioration des repas, due à de meilleures connaissances en matière culinaire, rendent l'alimentation africaine plus acceptable par les Blancs. Un nombre grandissant de familles noires adoptent de nouvelles pratiques, comme l'illustre la figure 4 qui représente un jeune enfant africain mangeant ce qui ressemble à un repas de couleur «brune » (vraisemblablement préparé à partir de petites céréales) dans un bol moderne. Certains citadins africains fusionnent les cuisines occidentale et africaine dans leur vie quotidienne. De plus, ces compétences dans le domaine de la cuisine occidentale ouvrent pour eux la perspective de trouver un emploi de cuisinier et d'aide domestique au sein des communautés blanches ${ }^{155}$.

\footnotetext{
visé de 3000 personnes, seules quelque 600 femmes africaines de divers cantons de la ville reçoivent officiellement des cours d'économie domestique dans les "Schools on Wheels».

150 Allaart L. K., «Education for nutrition... », art. cité, pp. 196-181. Also see Weinmann H., Agricultural development..., op. cit., p. 39.

151 Masenda A., The Food Production Committee..., op. cit., p. 112.

152 Wylie Diana (2001), Starving on a Full Stomach: Hunger and the Triumph of Cultural Racism in Modern South Africa, Charlottesville, University of Virginia Press, pp. 209-213.

153 Masenda A., The Food Production Committee..., op. cit., p. 14.

154 Schmidt Elizabeth (1996), Peasants, traders and wives: Shona women in the history of Zimbabwe, 1870-1939, Harare, Baobab press, p. 66.

155 Allaart L. K., « Education for nutrition... », art. cité, pp. 196-181.
} 
Figure $n^{\circ} 4$ : Portrait d'un enfant africain en train de manger dans les années $1950^{156}$

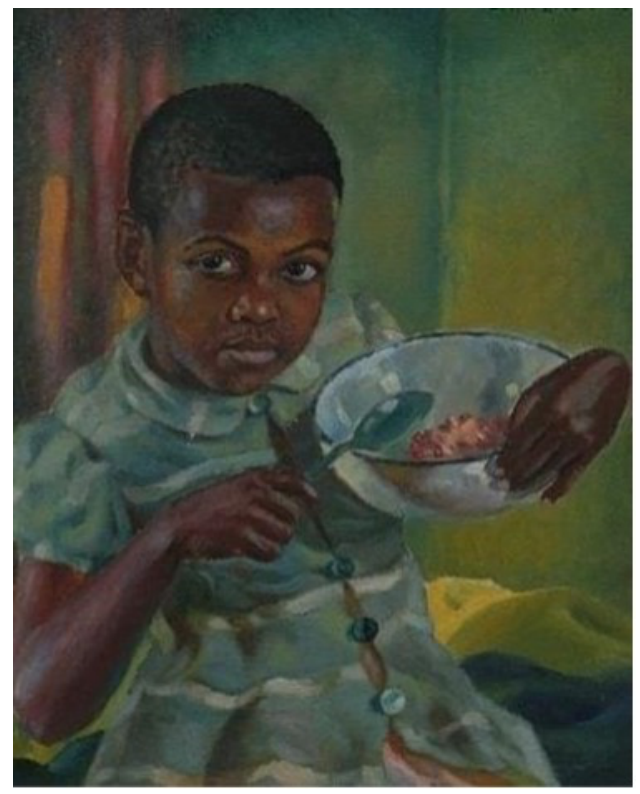

Dans le cadre plus large des débats sur l'histoire des citadins noirs au Zimbabwe et sur celle de la cuisine africaine, cet article s'est centré sur l'histoire sociale des petites céréales dans les familles africaines des années 1920 (élection du Gouvernement Responsable) au début des années 1950, après l'adoption de la Loi sur l'exploitation des terres (Land Husbandry Act) de 1951. Nous avons retracé la trajectoire complexe des pratiques culinaires liées aux petites céréales, en montrant comment des politiques explicites - qui portaient sur l'alimentation et le foncier - et des événements parfois imprévus - comme des sécheresses et des famines - ont contribué diversifier les méthodes de cuisine et l'alimentation des familles africaines citadines. Tout ceci est allé de pair avec des interactions croissantes entre Noirs et les Blancs dans les villes.

Cette étude s'est également intéressée aux mutations des relations sociales des familles noires, et à leur coexistence avec les communautés blanches et les autorités en milieu urbain, en particulier à Bulawayo. Les Africains ordinaires, notamment les femmes commerçantes, ont eux aussi exercé une influence en la matière, en contrôlant l'alimentation et les modes de préparation au sein d'espaces contestés où les choix agricoles des colons blancs étaient systématiquement privilégiés, comme cela a été le cas pour le maïs au détriment des petites céréales. Les Africains proches des Blancs ont adopté des idées et des méthodes culinaires nouvelles; d'autres ont continué à avoir recours à des méthodes dites traditionnelles de préparation et de consommation des aliments. II n'est pas rare qu'ils aient combiné les deux : méthodes traditionnelles et idées nouvelles des colons ont fusionné pour créer de nouvelles pratiques culinaires adaptées aux réalités sociales, économiques et culturelles des familles africaines.

156 Tiré de Sekai Nzenza, « Our disappearing traditional food », The Herald, 12 mai 2014, en ligne. URL : https://www.herald.co.zw/ourdisappearing-traditional-food// (Consulté le 18 juillet 2019). 
En examinant ces relations complexes entre l'État, les Africains et la nourriture, nous avons montré que, chez les Noirs comme chez les Blancs, la vision de la nourriture et des pratiques culinaires n'a été ni linéaire ni statique. Si le projet colonial visait à contrôler la maind'œuvre africaine, les lignes de fracture n'étaient pas complètement rigides entre Noirs et Blancs, et la communauté des colons n'a pas toujours été homogène face aux Africains ou aux modes de vie dits "traditionnels». Ces relations mouvantes ont eu un impact important sur I'histoire des petites céréales, mais aussi sur les conceptions de l'alimentation et du statut social par les Africains.

Bryan Kauma

Université de Stellenbosch (Afrique du Sud)

Sandra Swart

Université de Stellenbosch (Afrique du Sud)

\section{Bibliographie}

ALCOFf Linda Martin (2015), The Future of Whiteness, Malden, Polity Press.

AlLAART L. K. (1982), «Education for nutrition », The Central African Journal of Medicine, 28(8), pp. 195197.

AlvORD Emory D. (1948), «The progress of Native agriculture in Rhodesia », New Rhodesia, 15, pp. 132.

BARNES Teresa et WIN Everjoice (1992), To Live A Better Life: An Oral History of Women in the City of Harare, 1930-70, Harare, Baobab Books.

BARNES Teresa (1999), "We Women Worked So Hard": Gender, Urbanization and Social Reproduction in Colonial Harare, Zimbabwe, 1930-1956, Portsmouth, N.H. Heinemann.

BURKE Timothy (1996), Lifebouy Men, Lux Women: Commodification, consumption and cleanliness in modern Zimbabwe, Durham, Duke University Press.

CHIGODORA Joshua (1997), "Famine and Drought: The Question of Food Security in Zimbabwe», Drought Network News (1994-2001), pp. 32-40.

Chipembere Ennie (2007), Colonial urban policy and Africans in urban areas, with special focus on housing, Salisbury, 1929-1964, Thèse de doctorat en histoire, Université du Zimbabwe.

Coetzee Renata (1982), Funa and Food from Africa: Roots of traditional African food culture, Durban, Butterworths.

CUSACK Igor (2000), "African Cuisines: Recipes for Nation-Building? ", Journal of African Cultural Studies, 13(2), pp. 207-225.

DESMOND Judy (1963), Traditional Cookery in Southern Africa, Cape Town, Books of Africa.

DORO Elijah (2020), A socio-environmental history of commercial tobacco farming in Southern Rhodesia and Zimbabwe, 1893-2000, Thèse de doctorat en histoire, Université de Stellenbosch. 
DUBE Thembani (2015), Shifting identities and the transformation of the Kalanga people of Bulilimamangwe district, Matabeleland South, Zimbabwe, c. 1946-2005, Thèse de doctorat en histoire, Université du Witwatersrand.

Duggan William R. (1980), « The Native Land Husbandry Act of 1951 and the Rural African Middle Class of Southern Rhodesia ", African Affairs, 79, pp. 227-239.

FAIRBRIDGE Kingsley (1927), The autobiography of Kingsley Fairbridge, London, Oxford University Press.

GALE D.W. (1979), Rhodesia 1890-1970, Eighty Years onwards, Bulawayo, D.A. Blumberg.

GelfaNd Michael (1971), Diet and Tradition in African culture, Édimbourg et Londres, Livingstone ed.

Government OF SOUthern RHOdesiA (1950), Township Maps of Zimbabwe, Cape Town, University of Cape Town.

GUYER Jane (1987), Feeding African Cities: Studies in Regional Social History, Bloomington, Indiana University Press.-

HOWMAN Roger (1943), "The native labourer and his food", South African Institute of Race Relations (SAIRR).

JAYNE T. S. et RUBEY Lawrence (1998), « Maize milling, market reform and urban food security: The case of Zimbabwe », World Development, 21(6), pp. 975-988.

Kuper Hilda, Hughes A. J. B, van Alsen J (dir.) ([1954], 2017), The Shona and Ndebele of Southern Rhodesia, London, Routledge.

HARRIS Jessica (2010), The African cookbook: Tastes of a continent, New York, Simon and Schuster.

HASTORF Christine A. (2017), The Social Archaeology of Food: Thinking about Eating from Prehistory to the Present, Cambridge, Cambridge University Press.

Hove Godfrey (2015), The State, Farmers and Dairy Farming in Colonial Zimbabwe (Southern Rhodesia), c. 1890-1951, Thèse de doctorat en histoire, Université de Stellenbosch.

ILIFFE John (1987), The African Poor: A History, Cambridge, Cambridge University Press.

(1990), Famine in Zimbabwe, 1890-1960, Gweru, Mambo Press.

Jeater Diana (2020), «African Women in Colonial Settler Towns in East and Southern Africa », Oxford Research Encyclopaedia, African History, Oxford, Oxford University Press, pp. 1-27.

KAUMA Bryan (2021), " "Small grains, small gains": Small grains production and marketing in Southern Rhodesia", South African Historical Journal, en ligne. URL: https://www.tandfonline.com/doi/abs/10.1080/02582473.2021.1938192 (consulté le 15 septembre 2021).

KAYONGO-Male Diane et ONYANGo Philista (1984), The sociology of the African family, London, Longman.

LIVNeH Avital (1976), Precolonial polities in Southern Zambezia and their political communications», Thèse de doctorat, Université de Londres.

MACHINGAIDZE Victor (1980), The development of settler capitalist agriculture in Southern Rhodesia with particular reference to the role of the state, 1908-1939, Thèse de doctorat en histoire, Université de Londres. 
Madimu Tapiwa (2016), « Responsible Government and Miner-Farmer Relations in Southern Rhodesia, 1923-1945 », South African Historical Journal, 68(3), pp. 366-389.

MARAVANYIKA Simion (2012), "Local responses to colonial evictions, conservation and commodity policies among the Shangwe », African Nbula, 5, pp. 1-20.

MASUKU Fuller (1989), A study of agricultural change in the Ntabazinduna reserve with particular reference to the colonial period, 1923-1939, Mémoire de master, Université du Cap.

MASENDA A. (1987), The Food Production Committee and state food policy in colonial Zimbabwe during the 1940s, Mémoire de master, Université du Zimbabwe.

MAZARIRE Gerald (2009), «Reflections on pre-colonial Zimbabwe, c.850-1880s », in B. RAfTOPOULOS et A. MLAMBO (dir.), Becoming Zimbabwe: A History from the pre-colonial period to 2008, Harare, Weaver Press, pp. 1-38.

MCCANN James (2010), Stirring the pot: A history of African cuisine, Bloomsbury, Hurst and Co.

MeREDITH Martin (1979), The Past is Another Country: Rhodesia, Norfolk, Andre Deutsch.

MeRket-HeSs Kate (2016), The rural modern: Reconstructing the Self and State in Republican China, Chicago, University of Chicago Press.

Mostey Paul (1983), The Settler economies: Studies in the economic history of Kenya and Southern Rhodesia 1900-1963, Cambridge, Cambridge University Press.

Musemwa Muchapara (2014), Water History and politics in Zimbabwe: Bulawayo's struggles with the environment, 1894-2008, Trenton, Africa World Press.

National Research Council (1996), Lost Crops of Africa: Grains, Washington, Board on Science and Technology for International Development, National Academies Press.

NCUBE Sibanengi (2018), Colonial Zimbabwe Tobacco Industry: Global, regional and local relations, 1949-1979, Thèse de doctorat en histoire, Université du Free State.

NCUBE Trevor (1980), «Peasant production and marketing of grain crops in Zimbabwe, 1890-1980. An overview », Handerson Seminar Paper, 72.

NeHWATI Francis (1970), "The social and communal background to "Zhii": The African riots in Bulawayo, Southern Rhodesia in 1960 », African Affairs, 69, pp. 250-266.

NoBBS Eric (1906), «The mines as a market: The crops required for native diet », Rhodesian Agricultural Journal, 20, pp. 758-800.

NYATHI Pathisa (2020), African food, Bulawayo, Amagugu Publishers.

PAGE Sam L et PAGE Helena (1991), «Western hegemony over African agriculture in southern Rhodesia and its continuing threat to food security in independent Zimbabwe ", Agriculture and Human values, 8 , pp. 3-18.

Phimister lan (1974), «Peasant Production and Underdevelopment in Southern Rhodesia, 1890-1914 », African Affairs, 73, pp. 217-228.

PUNT Eira (1979), The development of African agriculture in Southern Rhodesia with particular reference to the interwar years, Mémoire de master en histoire, Université de Natal. 
Preben Kaarsholm (1995), "Si Ye Pambili-Which Way Forward?: Urban Development, Culture and Politics in Bulawayo », Journal of Southern African Studies, 21(2), pp. 225-245.

RANGer Terence (2010), Bulawayo Burning: The social history of a Southern African City, 1893-1960, Harare, Weaver.

Ruzivo Munetsi (2017), "Ecumenical initiatives in Southern Rhodesia: A history of the Southern Rhodesia Missionary Conference, 1903-1945 », Studia Historiae Ecclesiaaticae, 43(1), pp. 1-17.

SAMASUwo Nhamo (2003), "Food Production and War Supplies: Rhodesia's Beef Industry during the Second World War, 1939-1945 », Journal of Southern African Studies, 29(2), pp. 487-502.

SCHMIDT Elizabeth (1996), Peasants, traders and wives: Shona women in the history of Zimbabwe, 18701939, Harare, Baobab press.

SHutt Allison K. (2015), Manners Make a Nation: Racial Etiquette in Southern Rhodesia, 1910-1963, New York, University of Rochester Press.

SteELE Murray (1972), The foundations of native policy: Southern Rhodesia, 1923-1933, Thèse de doctorat en histoire, Université Simon Fraser.

STEEN Karin (2011), «A time to farm: A qualitative inquiry into the dynamics of the gender regime of land and labour rights in subsistence farming, an example from the Chiweshe communal area, Zimbabwe », Lund Dissertations in Sustainable Science, 2, pp. 45-51.

VAUGHAN Megan (1991), Curing their IIls: Colonial Power and African IIIness, California, Stanford University Press.

VERA Yvonne (1998), Butterfly burning, Harare, Baobab

VICKERY Kenneth (1985), "Saving Settlers: Maize Control in Northern Rhodesia », Journal of Southern African Studies, 11(2), pp. 212-234.

Weinmann H. (1972), Agricultural research and development in Southern Africa, 1890-1923, Salisbury, Université de Rhodésie

WeSt Michael O. (2002), The Rise of an African Middle Class: Colonial Zimbabwe, 1898-1965, Bloomington, Indiana University Press.

WHIT William (1999), « Soul Food as Cultural Creation », Journal for the Study of Food and Society, 3(1), pp. 37-47.

WiLliAMS-Forson Psyche (2014), " "I Haven't Eaten If I Don't Have My Soup and Fufu": Cultural Preservation through Food and Foodways among Ghanaian Migrants in the United States ", Africa Today, 61, pp. 68-87.

WILMOT Alexander (1895), The Story of the Expansion of Southern Africa, Londres, T. F. Unwin.

WYLIE Diana (2001), Starving on a Full Stomach: Hunger and the Triumph of Cultural Racism in Modern South Africa, Charlottesville, University of Virginia Press

YoSHIKUNI Tsuneo (2007), African Urban Experiences in Colonial Zimbabwe: A Social History of Harare before 1925, Oxford, African Books Collective.

YUdelman Montague (1964), Africans on the land, Cambridge, Harvard University Press. 